\title{
Judgement Aggregation in Search for the Truth
}

Citation for published version (APA):

Bozbay, I., Dietrich, F. K., \& Peters, H. J. M. (2011). Judgement Aggregation in Search for the Truth. METEOR, Maastricht University School of Business and Economics. METEOR Research Memorandum No. 040 https://doi.org/10.26481/umamet.2011040

Document status and date:

Published: 01/01/2011

DOI:

10.26481/umamet.2011040

Document Version:

Publisher's PDF, also known as Version of record

\section{Please check the document version of this publication:}

- A submitted manuscript is the version of the article upon submission and before peer-review. There can be important differences between the submitted version and the official published version of record.

People interested in the research are advised to contact the author for the final version of the publication, or visit the DOI to the publisher's website.

- The final author version and the galley proof are versions of the publication after peer review.

- The final published version features the final layout of the paper including the volume, issue and page numbers.

Link to publication

\footnotetext{
General rights rights.

- You may freely distribute the URL identifying the publication in the public portal. please follow below link for the End User Agreement:

www.umlib.nl/taverne-license

Take down policy

If you believe that this document breaches copyright please contact us at:

repository@maastrichtuniversity.nl

providing details and we will investigate your claim.
}

Copyright and moral rights for the publications made accessible in the public portal are retained by the authors and/or other copyright owners and it is a condition of accessing publications that users recognise and abide by the legal requirements associated with these

- Users may download and print one copy of any publication from the public portal for the purpose of private study or research.

- You may not further distribute the material or use it for any profit-making activity or commercial gain

If the publication is distributed under the terms of Article $25 \mathrm{fa}$ of the Dutch Copyright Act, indicated by the "Taverne" license above, 


\section{Maastricht University}

Irem Bozbay, Franz Dietrich, Hans Peters

J udgment aggregation in search for the truth

$\mathrm{RM} / 11 / 040$

\section{METEOR}

Maastricht University School of Business and Economics

Maastricht Research School of Economics

of Technology and Organization

P.O. Box 616

NL - 6200 MD Maastricht

The Netherlands 


\title{
Judgment aggregation in search for the truth
}

\author{
İrem Bozbay \\ Maastricht University \& LSE \\ Franz Dietrich \\ London School of Economics
}

\author{
Hans Peters \\ Maastricht University
}

June 2011 (minor revisions later)

\begin{abstract}
We analyse the problem of aggregating judgments over multiple issues from the perspective of efficient aggregation of voters' private information. While new in judgment aggregation theory, this perspective is familiar in a different body of literature about voting between two alternatives when voters' disagreements stem (fully or partly) from conflicts of information rather than interests. Combining the two literatures, we consider a simple judgment aggregation problem and model the private information underlying voters' judgments. We analyse the resulting strategic incentives and determine which voting rules lead to collective judgments that efficiently use all private information, assuming that voters share a preference for true collective judgments. We find that in many, but not all cases a quota rule should be used, which decides on each issue according to whether the proportion of 'yes' votes exceeds a particular quota.
\end{abstract}

Keywords: judgment aggregation, private information, efficient information aggregation, strategic voting

\section{Introduction}

In the by now well-established theory of judgment aggregation, a group needs to form a 'yes' or 'no' judgment on different issues, based on the judgments of the group members on these issues. For instance, the jury in a court trial might need to form judgments on whether the defendant has broken the contract, and whether the contract is legally valid; the United Nations security council might need to form judgments on whether country $\mathrm{X}$ is threatened by a military coup, and whether the economy of country $\mathrm{X}$ is collapsing; and so on. Group judgments matter in practice. They may determine group action: in the court trial example, they may determine whether the defendant is convicted, and in the United Nations example they may determine whether a large-scale international intervention in country $\mathrm{X}$ will happen.

So far, nearly the entire judgment aggregation theory follows the classical socialchoice theoretic approach of aiming to find out how - and whether - group judgments 
can reflect the individuals' judgments in a procedurally fair manner, where 'fair' is spelled out in terms of axiomatic conditions on the aggregation rule (such as the anonymity condition or the Pareto-type condition of respecting unanimous judgments). The recent Symposium on Judgment Aggregation in Journal of Economic Theory (C. List and B. Polak eds., 2010, vol. 145(2)) illustrates well this social-choice theoretic approach, as well as the state of the art of the theory, which we review below. This approach is certainly important in many contexts. It is nonetheless surprising that little attention is given to a different, 'epistemic' approach of aiming to track the truth, i.e., reach true group judgments. The theory does not model the private information underlying voters' judgments, thereby preventing itself from studying questions of efficient information aggregation. Yet such an epistemic perspective seems particularly natural in the context of aggregating judgments (rather than preferences ${ }^{1}$ ). In our court trial example, the ultimate goal seems indeed to be to find out independent facts (of whether the defendant has broken the contract and whether the contract is legally valid). So, the jury's voting rule should be optimised with respect to the goal that the resulting group judgments are true, not that they are fair to the jurors.

This alters the mechanism design problem altogether. Properties of voting rules standardly assumed in judgment aggregation theory, such as respecting unanimous judgments or anonymity, cannot be taken for granted anymore. If they turn out to be justified, they derive their justification from the truth-tracking goal rather than fairness considerations. To illustrate the contrast, suppose each juror expresses the judgment (opinion) that the contract was broken. A collective 'broken' judgment would then of course count as good from the classical social-choice theoretic perspective of procedural fairness. However, from a truth-tracking perspective, much depends on questions such as whether the jurors' judgments are sufficient evidence for breach of contract, and whether voters have expressed their judgments truthfully.

This paper analyses judgment aggregation from the truth-tracking and strategicvoting perspective. We model voters' private information, allowing us to ask questions about efficient information aggregation and strategic voting in a Bayesian voting game setting. Though new within judgment aggregation theory, this approach is wellestablished in a different body of literature about voting between two alternatives, which started with seminal work by Austen-Smith and Banks (1996) and Feddersen and Pesendorfer (1997) and can be placed in the broader context of work on the Condorcet Jury Theorem (see the review below). In the base-line case, voters share a common interest of finding out the 'correct' alternative, but hold possibly conflicting private information about which of the alternatives might be 'correct'. The voting rule should be designed so as to help finding the 'correct' alternative by making optimal use of all the private information scattered across the voters. So, the goal is efficient information aggregation. Such an 'epistemic' binary collective choice problem can in fact be viewed as a special judgment aggregation problem, involving just one issue. Our court trial example involves two issues: firstly, whether the contract was broken, and secondly, whether it is legally valid. If instead only the first issue were on the jury's agenda, the jury would face a single-issue judgment aggregation problem, or

\footnotetext{
${ }^{1}$ In preference aggregation theory, the core of social choice theory, an epistemic perspective would be less natural since there is no 'true preference' to be found.
} 
equivalently, a binary collective choice problem. The entire machinery and results of the mentioned binary collective choice literature could then be applied in order to design the voting rule.

This paper therefore combines two so far disconnected bodies of work, namely the judgment aggregation literature and the mentioned binary collective choice literature. We believe that these two literatures can learn from each other, and that a fruitful combination can help fill gaps in each of them. Indeed, it seems important that the former benefits from methodologies developed by the latter, and that the latter is extended beyond single-issue agendas towards more complex agendas with multiple issues. Analysing this multi-issue case does not reduce to analysing each issue separately, since preferences establish links between different issues.

It is worth starting simple. This paper therefore assumes that the group faces an agenda with just two issues, the simplest kind of multi-issue agenda; but many of our results generalize easily. Though simple, agendas with just two issues are important in practice. Our court trial example and United Nations example each involve two issues. To mention further two-issue agendas, a medical commission might need to issue joint judgments on whether a therapy is effective, and whether it is compatible with given ethical standards; members of a political party in charge of elaborating the party programme might seek joint judgments on whether a tax cut is affordable, and whether it is popular; a university hiring committee might seek joint judgments on whether a given candidate is good at research, and whether he or she is good at teaching; and finally, economic advisors to a government during the banking crisis in 2008 might need to issue collective judgments on whether a given bank has short-term liquidity problems, and whether it has long-term liquidity problems.

The issues of an agenda could in principle be mutually interconnected, so that the judgments taken on the issues logically constrain each other; for instance, a 'no' judgment on all issues might be inconsistent. Indeed, interconnections are what render judgment aggregation non-trivial if the usual social-choice theoretic approach of procedural fairness is taken. ${ }^{2}$ However, within our truth-tracking approach, mechanism design is non-trivial even if the issues are mutually independent. We therefore assume independence between issues, leaving the case of interconnections to future research.

Structure of the paper. Section 2 introduces our model, in which voters vote on the basis of private information and are guided by 'truth-tracking preferences', i.e., aim for true collective judgments. Section 3 addresses the key question of how to design the voting rule such that it leads to efficient decisions as well as simple-minded, truthful voting behaviour in equilibrium. It will turn out that in many, but not all cases one should use a 'quota rule', which decides on each issue according to whether the number of 'yes' judgments on the issue exceeds a particular quota. The details depend on the exact kind of truth-tracking preferences, i.e., whether preferences are 'simple' or 'consequentialist' in a sense defined below. Section 4 analyses the notion of truthful behaviour, by determining the conditions under which a 'sincere' voter

\footnotetext{
${ }^{2}$ In the absence of interconnections one can safely aggregate by taking a separate vote on each issue. This never generates inconsistent collective judgments and meets all standard social-choice theoretic requirements such as anonymity.
} 
directly reveals his information in his vote. Finally, the appendix contains all proofs.

Literature review. We now selectively review the two literatures to which this paper connects, beginning with judgment aggregation theory. As mentioned, this theory's primary objective has so far been to find out which voting rules can aggregate the judgments of group members over some issues in accordance with certain axiomatic requirements with a classic social-choice theoretic flavour, such as unanimity preservation (the counterpart of the Pareto principle) and independence (the counterpart of Arrow's independence of irrelevant alternatives). A series of possibility and impossibility results successfully address this query, by giving answers which depend, firstly, on the axiomatic requirements on the voting rule, and secondly, on the agenda of issues under consideration (e.g., List and Pettit 2002, Dietrich 2006, 2007, 2010, Nehring and Puppe 2008, 2010, Dietrich and List 2007a, 2008, Dokow and Holzman 2010a, 2010b, Dietrich and Mongin 2010; see also precursor results by Guilbaud 1952 and Wilson 1975; for an introductory overview see List and Polak 2010). By contrast, a small minority of papers about judgment aggregation take a truth-tracking perspective (e.g., Bovens and Rabinowicz 2006 and List 2005). Their innovation is to apply the classical Condorcet Jury Theorem to judgment aggregation. Despite taking a truth-tracking perspective, they have little in common with our work, since private information and strategic incentives are not being considered. ${ }^{3}$ List and Pettit (2011) provide the most systematic philosophical analysis of the truth-tracking approach, already discussing strategic incentives and private information and drawing on the second body of literature to which we now turn.

As for this second body of literature, it is concerned with voting rules for binary choice problems in which disagreements are driven (partly or totally) by conflicting information rather than conflicting interests. Specifically, the utilities which voters derive from decisions are affected by the same unknown 'state of the world', about which voters have private information. Austen-Smith and Banks (1996) and Feddersen and Pesendorfer (1997) show that it typically cannot be rational for all voters to vote sincerely, and that the choice of voting rule matters considerably for sincere voting and efficient information aggregation. While the former authors consider the 'purely epistemic' case without conflict of interest, the latter authors introduce some preference heterogeneity (and focus primarily on large electorates). AustenSmith and Feddersen $(2005,2006)$ add an extra dimension of pre-voting deliberation. Duggan and Martinelli (2001) extend the approach to continuous rather than binary private information. Feddersen and Pesendorfer (1998), Coughlan (2000) and Gerardi (2000) examine the (in)effectiveness of unanimity rule in 'protecting the innocent' in jury trials. Goertz and Maniquet (2011) analyse efficient information aggregation in large electorates, showing that approval voting outperforms other voting rules in their setting.

\footnotetext{
${ }^{3}$ Dietrich and List (2007b) analyse strategic voting in judgment aggregation, but in a sense not relevant to us since strategic voting is not modelled as coming from private information and a voter is motivated by the somewhat different goal that the collective judgments match his own judgments. Such assumptions are more natural under common knowledge of each other's judgments than under informational asymmetry. See also related work by Nehring and Puppe (2002, 2007).
} 


\section{The Model}

\subsection{A simple judgment aggregation problem}

We consider a group of voters, labelled $i=1, \ldots, n$, where $n \geq 2$. This group needs a collective judgment on whether some proposition $p$ or its negation $\bar{p}$ is true, and whether some other proposition $q$ or its negation $\bar{q}$ is true. In our court trial example, $p$ states that the contract was broken, and $q$ that it is legally valid; in our job candidate example, $p$ states that the candidate is good at research, and $q$ that he or she is good at teaching; and so on for our other examples. The four possible judgment sets are $\{p, q\},\{p, \bar{q}\},\{\bar{p}, q\}$ and $\{\bar{p}, \bar{q}\}$; we abbreviate them by $p q, p \bar{q}, \bar{p} q$ and $\bar{p} \bar{q}$, respectively. For instance, $p \bar{q}$ means accepting $p$ but not $q$. Each voter votes for a judgment set in $\mathcal{J}=\{p q, p \bar{q}, \bar{p} q, \bar{p} \bar{q}\}$. After all voters cast their votes, a collective decision in $\mathcal{J}$ is taken using a voting rule. Formally, a voting rule is a function $f: \mathcal{J}^{n} \rightarrow \mathcal{J}$, mapping each voting profile $\mathbf{v}=\left(v_{1}, \ldots, v_{n}\right)$ to a decision $d \equiv f(\mathbf{v})$. Among the various voting rules, quota rules stand out as particularly natural and common. A quota rule is given by two thresholds $m_{p}, m_{q} \in\{0,1, \ldots, n+1\}$, and for each voting profile it accepts $p$ $[q]$ if and only if at least $m_{p}\left[m_{q}\right]$ voters accept it in the profile. Quota rules have three salient properties:

- Anonymity: For all voting profiles $\left(v_{1}, \ldots, v_{n}\right) \in \mathcal{J}^{n}$ and all permutations $\left(i_{1}, \ldots\right.$, $\left.i_{n}\right)$ of the voters, $f\left(v_{i_{1}}, \ldots, v_{i_{n}}\right)=f\left(v_{1}, \ldots, v_{n}\right)$. Informally, the voters are treated equally.

- Monotonicity: For all voting profiles $\mathbf{v}, \mathbf{v}^{\prime} \in \mathcal{J}^{n}$, if for each $r$ in $f(\mathbf{v})$ the voters who accept $r$ in $\mathbf{v}$ also accept $r$ in $\mathbf{v}^{\prime}$, then $f\left(\mathbf{v}^{\prime}\right)=f(\mathbf{v})$. Informally, additional support for the collectively accepted propositions never reverses the collective acceptance of these propositions.

- Independence: The decision on each proposition $r \in\{p, q\}$ only depends on the votes on $r .^{4}$ Informally, the group in effect takes two separate votes, one between $p$ and $\bar{p}$ and one between $q$ and $\bar{q}$.

Remark 1 A voting rule $f: \mathcal{J}^{n} \rightarrow \mathcal{J}$ is a quota rule if and only if it is anonymous, monotonic and independent.

We briefly sketch the proof of the non-trivial direction of implication. As can be shown, if a voting rule $f: \mathcal{J}^{n} \rightarrow \mathcal{J}$ is anonymous and independent, then it is given by two sets $M_{p}, M_{q} \subseteq\{0,1, \ldots, n\}$, in the sense that for each voting profile $\mathbf{v} \in \mathcal{J}^{n}$ the decision $f(\mathbf{v})$ contains $r(\in\{p, q\})$ if and only if the number of votes in $\mathbf{v}$ containing $r$ belongs to $M_{r}$. If $f$ is moreover monotonic, each set $M_{r}$ can be shown to take the form $\left\{m_{r}, m_{r}+1, \ldots, n\right\}$ for some threshold $m_{r} \in\{0,1, \ldots, n+1\}$. Clearly, $f$ is the quota rule with thresholds $m_{p}$ and $m_{q}$.

\footnotetext{
${ }^{4}$ Given a voting profile $\mathbf{v}$, the subprofile with respect to $r$ is denoted $\mathbf{v}_{r}\left(\in\{r, \bar{r}\}^{n}\right)$, and the collective decision with respect to $r$ is denoted $f_{r}(\mathbf{v})(\in\{r, \bar{r}\})$. Independence means that for all voting profiles $\mathbf{v}, \mathbf{v}^{\prime} \in \mathcal{J}^{n}$, if $\mathbf{v}_{r}=\mathbf{v}_{r}^{\prime}$, then $f_{r}(\mathbf{v})=f_{r}\left(\mathbf{v}^{\prime}\right)$.
} 


\subsection{A common preference for true collective judgments}

Exactly one judgment set in $\mathcal{J}$ is 'correct', i.e., contains propositions which are factually true. It is called the state (of the world) and is generically denoted by $s$. For instance, the state might be $p \bar{q}$, so that $p$ and $\bar{q}$ are true (and $\bar{p}$ and $q$ are false). Voters have identical preferences, captured by a common utility function $u: \mathcal{J} \times \mathcal{J} \rightarrow \mathbb{R}$ which maps any decision-state pair $(d, s)$ to its utility $u(d, s)$. Given voters' truthtracking goal, one would expect $u(d, s)$ to be high if $d=s$, i.e., if the decision is correct. But how exactly should $u$ be specified? We focus on two natural kinds of preferences:

Simple preferences. Here, the utility function is given by

$$
u(d, s)= \begin{cases}1 & \text { if } d=s \text { (correct decision) } \\ 0 & \text { if } d \neq s \text { (incorrect decision) }\end{cases}
$$

Such preferences are the simplest candidate for truth-tracking preferences. Correct decisions are preferred to incorrect ones, without further sophistication.

Consequentialist preferences. Here, we assume that the decision leads to one of two possible consequences, typically representing group actions. This is captured by a consequence function Co which maps the set of possible decisions $\mathcal{J}$ to a twoelement set of possible consequences. The consequence function might look as follows in examples given earlier. In our court trial example, the court decision $p q$ leads to conviction, since both premises of guilt are found to be satisfied $(\mathrm{Co}(p q)=$ 'conviction'), while the other decisions all lead to acquittal $(\operatorname{Co}(\bar{p} \bar{q})=\operatorname{Co}(p \bar{q})=\operatorname{Co}(\bar{p} q)=$ 'acquittal'). In our job candidate example, the decision $p q$ leads to a hire since the candidate is seen as meeting both criteria $(\mathrm{Co}(p q)=$ 'hire'), while the other decisions all lead to no hire $(\operatorname{Co}(\bar{p} \bar{q})=\operatorname{Co}(p \bar{q})=\operatorname{Co}(\bar{p} q)=$ 'no hire'). In our United Nations example, the decisions $p \bar{q}$ and $\bar{p} q$ each lead to a large-scale international intervention in country $\mathrm{X}(\mathrm{Co}(p \bar{q})=\operatorname{Co}(\bar{p} q)=$ 'intervention'), whereas the decisions $p q$ and $\bar{p} \bar{q}$ both lead to no intervention since the United Nations then consider an intervention as being too risky or unnecessary, respectively $(\mathrm{Co}(p q)=\mathrm{Co}(\bar{p} \bar{q})=$ 'no intervention'). In our bank rescuing example, the decisions $p \bar{q}$ and $\bar{p} q$ each lead to a governmental rescue plan for the bank $(\operatorname{Co}(p \bar{q})=\operatorname{Co}(\bar{p} q)=$ 'rescue'), whereas the decisions $p q$ and $\bar{p} \bar{q}$ both lead to no rescue plan since a rescue is seen as infeasible or unnecessary, respectively $(\mathrm{Co}(p q)=\operatorname{Co}(\bar{p} \bar{q})=$ 'no rescue'). The consequentialist utility function is given by

$$
u(d, s)= \begin{cases}1 & \text { if } \operatorname{Co}(d)=\operatorname{Co}(s) \text { (correct consequence) } \\ 0 & \text { if } \operatorname{Co}(d) \neq \operatorname{Co}(s) \text { (incorrect consequence) }\end{cases}
$$

Incorrect decisions $(d \neq s)$ can have correct consequences $(\operatorname{Co}(d)=\operatorname{Co}(s))$. The hiring committee might view the candidate as good at research and bad at teaching when in fact the opposite is true, so that the resulting consequence ('no hire') is correct for wrong reasons. This gives high utility under consequentialist preferences, but low utility under simple preferences. ${ }^{5}$

\footnotetext{
${ }^{5}$ In the judgment aggregation literature, the two possible consequences are usually represented by
} 


\subsection{Private information and strategies}

If voters had not just common preferences, but also common information about what the state might be, then no disagreement could arise. We however allow for informational asymmetry. Each voter has a type, representing private information or evidence. $^{6}$ A voter's type takes the form of an element of $\mathcal{J}$, generically denoted by $t$. For instance, a voter of type $t=p \bar{q}$ has evidence for $p$ and for $\bar{q}$. We write $\mathbf{t}=\left(t_{1}, \ldots, t_{n}\right) \in \mathcal{J}^{n}$ for a profile of voters' types. Nature draws a state-types combination $(s, \mathbf{t}) \in \mathcal{J}^{n+1}$ according to a probability measure denoted Pr. When a proposition $r \in\{p, \bar{p}, q, \bar{q}\}$ is meant to represent part of voter $i$ 's type rather than part of the true state, we often write $r_{i}$ for $r$. For instance, $\operatorname{Pr}\left(p_{i} \mid p\right)$ is the probability that voter $i$ has evidence for $p$ given that $p$ is true. By assumption, the prior probability that $r(\in\{p, \bar{p}, q, \bar{q}\})$ is true is denoted

$$
\pi_{r}=\operatorname{Pr}(r)
$$

and belongs to $(0,1)$, and the probability of getting evidence for $r$ given that $r$ is true is denoted

$$
a_{r}=\operatorname{Pr}\left(r_{i} \mid r\right),
$$

belongs to $(1 / 2,1)$, and does not depend on the voter $i$. The parameters $a_{p}, a_{\bar{p}}, a_{q}, a_{\bar{q}}$ measure the reliability of private information, as they represent probabilities of receiving 'truth-telling' information. The lower bound of $1 / 2$ reflects the (standard) idea that information is more reliable than a fair coin.

By assumption, voters' types are independent conditional on the state, and in addition the state and the types w.r.t. $p$ are independent of the state and the types w.r.t. $q .^{7}$ These independence assumptions allow one to express the joint distribution of the state and the types by just a few parameters, namely $\pi_{p}, \pi_{q}, a_{p}, a_{\bar{p}}, a_{q}, a_{\bar{q}}$. For instance, the probability that the state is $p q$ and all voters receive the truth-telling evidence $p q$ is

$$
\operatorname{Pr}\left(p q, p_{1} q_{1}, p_{2} q_{2}, \ldots, p_{n} q_{n}\right)=\operatorname{Pr}(p q) \operatorname{Pr}\left(p_{1} q_{1}, p_{2} q_{2}, \ldots, p_{n} q_{n} \mid p q\right)=\pi_{p} \pi_{q} a_{p}^{n} a_{q}^{n} .
$$

Each voter submits a vote in $\mathcal{J}$ based on his type. A (voting) strategy is a function $\sigma: \mathcal{J} \rightarrow \mathcal{J}$, mapping each type $t \in \mathcal{J}$ to the type's vote $v=\sigma(t)$. We write $\boldsymbol{\sigma}=\left(\sigma_{1}, \ldots, \sigma_{n}\right)$ for a profile of voters' strategies. Together with a voting rule $f$ and a common utility function $u$, we now have a well-defined Bayesian game.

For a given type profile $\mathbf{t} \in \mathcal{J}^{n}$, we call a decision $d$ efficient if it has maximal expected utility conditional on the full information $\mathbf{t} .^{8}$ Some common notions of voting behaviour can now be adapted to our framework:

two conclusion propositions, $c$ and $\bar{c}$. In our first two examples, the consequence function is encoded in the biconditional $c \leftrightarrow(p \wedge q)$, whereas in our last two examples it is encoded in the biconditional $c \leftrightarrow((p \wedge q) \vee(\bar{p} \wedge \bar{q}))$

${ }^{6}$ The type could represent information that is not shared with other voters because of a lack of deliberation or limits of deliberation. More generally, a voter $i$ 's type could represent uncertainty of other voters about $i$ 's beliefs.

${ }^{7}$ Recall that the state consists of a proposition in $\{p, \bar{p}\}$ and another in $\{q, \bar{q}\}$. The first [second] of these propositions is what we call the state w.r.t. $p[q]$. A voter's type w.r.t. $p[q]$ is defined similarly.

${ }^{8}$ I.e., $d$ maximizes $E(u(d, S) \mid \mathbf{t})=\sum_{s \in \mathcal{J}} u(d, s) \operatorname{Pr}(s \mid \mathbf{t})$, where ' $S$ ' denotes the random variable generating the state $s$ in $\mathcal{J}$. 
- A strategy $\sigma$ of a voter is informative if $\sigma(t)=t$ for all types $t$. An informative voter directly reveals his information in his vote.

- A strategy $\sigma$ of a voter is sincere if for every type $t$, the vote $\sigma(t)$ maximises the expected utility conditional on the information $t$. A sincere voter votes for the decision which maximises the expected utility conditional on his type; so, he acts as if his vote alone determined the decision, neglecting the other voters and their strategies. Technically, this amounts to optimal behaviour in a hypothetical single-player decision problem.

- A strategy profile $\boldsymbol{\sigma}=\left(\sigma_{1}, \ldots, \sigma_{n}\right)$ is rational if each strategy is a best response to the other strategies, i.e., if the profile is a Nash equilibrium of the corresponding Bayesian game. Hence, each voter maximises the expected utility of the collective decision given the strategies of the other voters. (In this maximisation exercise, it turns out that a voter must only consider cases in which his vote is pivotal. Under a quota rule with majority thresholds, a voter is for instance pivotal if half of the other voters votes $p q$ and the other half votes $\bar{p} \bar{q}$.)

- A strategy profile $\boldsymbol{\sigma}=\left(\sigma_{1}, \ldots, \sigma_{n}\right)$ is efficient if for every type profile $\mathbf{t}=$ $\left(t_{1}, \ldots, t_{n}\right)$ the resulting decision $d=f\left(\sigma_{1}\left(t_{1}\right), \ldots, \sigma_{n}\left(t_{n}\right)\right)$ is efficient (i.e., has maximal expected utility conditional on full information $\mathbf{t}$ ). Hence, all the information spread across the group is used efficiently: the collective decision is no worse than a decision of a (virtual) social planner who has full information.

While informativeness and sincerity are properties of a single strategy (or voter), rationality and efficiency refer to an entire profile.

Finally, to avoid distraction by special cases, we make two assumptions. First, we exclude the degenerate case in which some decision in $\mathcal{J}$ is not efficient for any type profile whatsoever. Second, we exclude efficiency ties, i.e., we exclude those special parameter combinations such that some type profile $\mathbf{t}$ leads to different efficient decisions (with different consequences when we assume consequentialist preferences).

\section{Which voting rules lead to efficient information ag- gregation?}

\subsection{Setting the stage}

Our objective is to design the voting rule ('mechanism') in such a way as to yield efficient decisions on the basis of informative votes. In short, the voting rule should render informative voting efficient. ${ }^{9}$ We begin by justifying this objective. Prima facie, two goals are of interest. The rule should, firstly, lead to efficient outcomes, and, secondly, encourage simple-minded, truthful behaviour. By such behaviour we mean informative voting. ${ }^{10}$ To reach the second goal, informative voting should be

\footnotetext{
${ }^{9}$ By saying "informative voting" without referring to a particular voter, we mean "informative voting by all voters".

${ }^{10}$ One might alternatively mean sincere voting - but in practice there is little difference, since informative and sincere voting coincide under reasonable informational assumptions. As one can show, if informative voting is not sincere, then there exists a decision $d \in \mathcal{J}$ such that no voter
} 
rational, i.e., occur in equilibrium. If informative voting is not just rational, but also efficient, both goals are reached. So, the double-goal is that informative voting be efficient and rational. Crucially, as we now show, whenever informative voting is efficient, it is a fortiori also rational - which explains our primary objective that informative voting be efficient.

Theorem 1 Consider an arbitrary common utility function $u: \mathcal{J}^{2} \rightarrow \mathbb{R}$.

(a) For any voting rule, if a strategy profile is efficient, then it is rational.

(b) There is an anonymous voting rule for which informative voting is efficient (hence, rational).

This theorem is general in that it applies to any kind of (common) preferences. The converse of part (a) does not hold: for instance, a constant voting rule makes all strategy profiles rational, but typically not efficient. The message of part (b) is positive but so far vague: it is always possible to make informative voting efficient (and rational), but apart from anonymity we do not know anything about the kind of voting rule we can use. And indeed, for some kinds of common preferences, it may not be possible to aggregate in an independent or monotonic way (as counterexamples show). But, once we narrow down to simple or consequentialist preferences, can - or even must - we aggregate in a monotonic resp. independent way? When can - or even must - we use a quota rule? Such questions are answered below.

\subsection{Simple preferences}

This section addresses the case of simple preferences, given by the common utility function (1). Which rules render informative voting efficient (hence, rational)? The answer is 'simple', as we will see. To state our result, we first define two coefficients: ${ }^{11}$

$$
\begin{aligned}
& k_{p}:=\min \left\{k \in\{0,1, \ldots, n+1\}: \frac{\pi_{p}}{1-\pi_{p}}>\left(\frac{1-a_{\bar{p}}}{a_{p}}\right)^{k}\left(\frac{a_{\bar{p}}}{1-a_{p}}\right)^{n-k}\right\}, \\
& k_{q}:=\min \left\{k \in\{0,1, \ldots, n+1\}: \frac{\pi_{q}}{1-\pi_{q}}>\left(\frac{1-a_{\bar{q}}}{a_{q}}\right)^{k}\left(\frac{a_{\bar{q}}}{1-a_{q}}\right)^{n-k}\right\} .
\end{aligned}
$$

These coefficients have an interpretation: as can be proved, for $p[q]$ to be more probably true than false given all information, at least $k_{p}\left[k_{q}\right]$ individuals need to receive evidence for $p[q]$, i.e., need to have a type containing $p[q]$.

Theorem 2 Assume simple preferences. Informative voting is efficient if and only if $f$ is the quota rule with the thresholds $k_{p}$ and $k_{q}$.

ever finds himself in an informational position to consider $d$ as best - a rather uninteresting, if not unnatural scenario.

${ }^{11}$ The minimum defining $k_{p}$ or $k_{q}$ should be interpreted as $n+1$ if the set whose minimum is being taken is empty. In fact, emptiness is impossible under simple preferences. This follows from our non-degeneracy assumption on the model parameters (which also implies that $k_{p}, k_{q} \in\{1, \ldots, n\}$ ). Note that in (3) and (4) the right hand side of the inequality is strictly decreasing in $k$. 
This result shows that the quota rule with thresholds $k_{p}$ and $k_{q}$ is the only rule we may use in view of making informative voting efficient (hence, rational). This result is much more specific than the purely existential claim in part (b) of Theorem 1. This progress was possible by focusing on simple preferences.

\subsection{Consequentialist preferences: first type}

We now turn to consequentialist preferences. Much depends on the nature of the consequence function. In principle, there exist $2^{4}=16$ potential consequence functions from $\mathcal{J}$ to a binary set of consequences. But, as we shall see shortly, there are only two non-degenerate consequence functions up to isomorphism. We therefore define two types of consequentialist functions. Consequentialist preferences (or the consequence function) are said to be:

- of type 1 if $\operatorname{Co}(p q)=\operatorname{Co}(\bar{p} \bar{q}) \neq \operatorname{Co}(p \bar{q})=\operatorname{Co}(\bar{p} q)$;

- of type 2 if $\operatorname{Co}(p q) \neq \operatorname{Co}(\bar{p} \bar{q})=\operatorname{Co}(p \bar{q})=\operatorname{Co}(\bar{p} q)$.

Our first two examples of consequentialist preferences in Section 2.2 are of type 1 , while our last two examples are of type 2 . But why are all non-degenerate consequences of one of these two types? Firstly, consequence functions for which each decision in $\mathcal{J}$ has the same consequence are of course degenerate and therefore uninteresting. Also consequence functions which depend only on the decision between $p$ and $\bar{p}$, or only on the decision between $q$ and $\bar{q}$, are degenerate, since in this case we are essentially back to a decision problem with a single proposition-negation pair, which has already been studied in the literature. ${ }^{12}$ The non-degenerate consequence functions are those which genuinely depend on both propositions. Among all of them, some assign each consequence to exactly two decisions in $\mathcal{J}$, while the others assign one consequence to three decisions and the other consequence to just one decision. As one can show, the former consequence functions are of type 1, while the latter are of type 2 up to isomorphism (i.e., up to exchanging $p$ and $\bar{p}$ and/or exchanging $q$ and $\bar{q})$. Thus, by studying our two types of consequence functions, we will have covered non-degenerate consequentialist preferences exhaustively.

We now address the first type, while the next subsection turns to the second type. One might at first expect there to be little resemblance between the current preferences and simple preferences in terms of the appropriate voting rule. For instance, even when all individuals have type $p q$, so that there is overwhelming evidence for state $p q$, the current preferences allow us to efficiently decide for $\bar{p} \bar{q}$, since this decision has the same consequence as $p q$. Surprisingly, despite the differences, consequentialist preferences of type 1 come much closer to simple preferences than to consequentialist preferences of type 2 in terms of mechanism design. The coefficients $k_{p}$ and $k_{q}$, defined earlier for simple preferences, again play a key role.

Theorem 3 Assume consequentialist preferences of type 1. A voting rule $f$ makes informative voting efficient and is monotonic if and only if it is the quota rule with thresholds $k_{p}$ and $k_{q}$.

\footnotetext{
${ }^{12}$ For instance, our UN intervention example would be degenerate if the question of whether to intervene only depended on whether the country is considered as being threatened by a military coup $(p$ or $\bar{p})$. The other pair of propositions $(q$ or $\bar{q}$ ) could then be eliminated from the voting process.
} 
So, as for simple preferences, the social planner is led to impose a quota rule with the particular thresholds $k_{p}$ and $k_{q}$. What distinguishes Theorem 3 from Theorem 2 is, for one, its somewhat different (and longer) proof, and secondly, the additional monotonicity requirement. Without this extra condition, a number of other voting rules become possible:

Corollary 1 Assume consequentialist preferences of type 1. A voting rule $f$ makes informative voting efficient if and only if for every voting profile $\mathbf{v} \in \mathcal{J}^{n}$ the decision $f(\mathbf{v})$ has the same consequence as the decision under the quota rule with thresholds $k_{p}$ and $k_{q}$ (i.e., Co $\circ=\mathrm{Co} \circ g$, where $g$ is this quota rule).

So, once we drop the monotonicity requirement, there is not just one possible voting rule, as for simple preferences, but $2^{4^{n}}$ possible rules (since there are 2 allowed decisions for each of the $4^{n}$ profiles in $\mathcal{J}^{n}$ ).

\subsection{Consequentialist preferences: second type}

We now turn to consequentialist preferences of type 2. The space of aggregation possibilities is somewhat different here. As we shall show, quota rules are not always possible, and when they are, the two thresholds must be calculated differently.

For all $k, l \in \mathbb{R}$, we define the coefficient

$$
\beta(k, l)=\frac{\pi_{p} a_{p}^{k}\left(1-a_{p}\right)^{n-k}}{\pi_{p} a_{p}^{k}\left(1-a_{p}\right)^{n-k}+\pi_{\bar{p}} a_{\bar{p}}^{n-k}\left(1-a_{\bar{p}}\right)^{k}} \times \frac{\pi_{q} a_{q}^{l}\left(1-a_{q}\right)^{n-l}}{\pi_{q} a_{q}^{l}\left(1-a_{q}\right)^{n-l}+\pi_{\bar{q}} a_{\bar{q}}^{n-l}\left(1-a_{\bar{q}}\right)^{l}} .
$$

One can show that $\beta(k, l)$ has a natural interpretation if $k, l \in\{0,1, \ldots, n\}$ : it is the probability that the state is $p q$ conditional on having $k$ times evidence for (and $n-k$ times evidence against) $p$ and $l$ times evidence for (and $n-l$ times evidence against) $q$. So, $\beta(k, l)=\operatorname{Pr}(p q \mid \mathbf{t})$ for some (hence, any) type profile $\mathbf{t} \in \mathcal{J}^{n}$ containing $p$ exactly $k$ times and $q$ exactly $l$ times; or equivalently,

$$
\beta(k, l)=\operatorname{Pr}\left(p \mid p_{1}, \ldots p_{k}, \bar{p}_{k+1}, \ldots, \bar{p}_{n}\right) \times \operatorname{Pr}\left(q \mid q_{1}, \ldots, q_{l}, \bar{q}_{l+1}, \ldots, \bar{q}_{n}\right) .
$$

As one can prove by drawing on the definition of the consequence function, given a type profile $\mathbf{t}$ containing $p$ exactly $k$ times and $q$ exactly $l$ times, if $\beta(k, l)>1 / 2$ then only the decision $p q$ is efficient, while otherwise the three other decisions are all efficient. This implies a first, simple characterization result. Henceforth, the number of votes for a proposition $r$ in a voting profile $\mathbf{v}$ is written $n_{r}^{\mathbf{v}}$.

Proposition 1 Assume consequentialist preferences of type 2. A voting rule $f$ makes informative voting efficient if and only if for every voting profile $\mathbf{v} \in \mathcal{J}^{n}$ the decision $f(\mathbf{v})$ is $p q$ if $\beta\left(n_{p}^{\mathbf{v}}, n_{q}^{\mathbf{v}}\right)>1 / 2$ and in $\{p \bar{q}, \bar{p} q, \bar{p} \bar{q}\}$ otherwise.

Which possibilities - if any - are left if we require the rule to be a quota rule? We begin by introducing two coefficients. Given that all voters hold evidence for $q$, how

many voters with evidence for $p$ does it minimally take for the decision $p q$ to become efficient? Similarly, given that all voters hold evidence for $p$, how many voters with 
evidence for $q$ does it take for the decision $p q$ to become efficient? The answer to these questions is given by the following numbers, respectively: ${ }^{13}$

$$
\begin{aligned}
& l_{p}:=\min \{k \in\{0, \ldots, n\}: \beta(k, n)>1 / 2\} \\
& l_{q}:=\min \{k \in\{0, \ldots, n\}: \beta(n, k)>1 / 2\} .
\end{aligned}
$$

Theorem 4 Assume consequentialist preferences of type 2. There exists a quota rule making informative voting efficient if and only if $\beta\left(l_{p}, l_{q}\right)>1 / 2$. In this case, that quota rule is unique and has the thresholds $l_{p}$ and $l_{q}$.
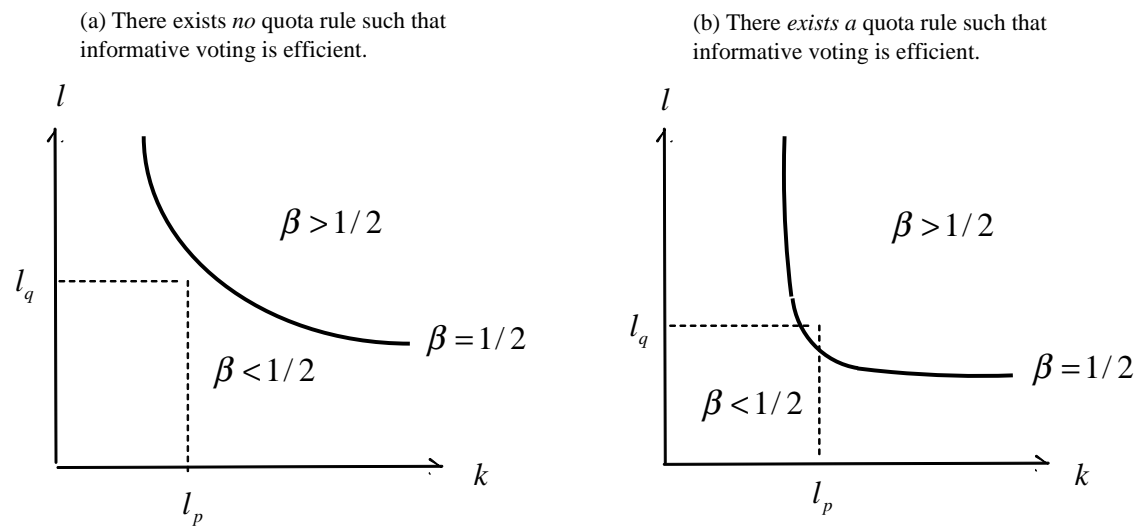

Figure 1: The function $\beta$

Figure $1 \mathrm{~b}$ illustrates the region to which $\left(l_{p}, l_{q}\right)$ must belong for a quota rule to be available. Unlike when preferences are simple or consequentialist of type 1 , and unlike in the classic literature for a single pair of propositions $p, \bar{p}$, we have a partial impossibility:

Corollary 2 Assume consequentialist preferences of type 2. For some, but not all combinations of values of the model parameters $\left(\pi_{p}, \pi_{q}, a_{p}, a_{\bar{p}}, a_{q}, a_{\bar{q}}\right.$ and $\left.n\right)$, there exists a quota rule making informative voting efficient.

For instance, if $\pi_{p}=\pi_{q}=0.5, a_{p}=a_{q}=a_{\bar{p}}=a_{\bar{q}}=0.7$ and $n=3$, no quota rule makes informative voting efficient, whereas if instead $\pi_{p}=\pi_{q}=0.6$, the quota rule with thresholds $l_{p}=l_{q}=2$ makes informative voting efficient.

While by Corollary 2 it may be utopian to aim for a full-fledged quota rule, we now show that one can always achieve two characteristic properties of quota rules, namely anonymity and monotonicity, while often losing the third characteristic property, namely independence. Specifically, we characterize the class of all monotonic and anonymous (but not necessarily independent) aggregation possibilities. As we shall see, this class consists of so-called quota rules 'with exception'. Such rules behave

\footnotetext{
${ }^{13}$ These two minima are taken over non-empty sets of values of $k$ (by the non-degeneracy assumption at the end of Section 2.3).
} 
like a quota rule as long as the profile does not fall into an 'exception domain', while generating the 'exception decision' $p q$ on the exception domain. Formally, a quota rule with exception $f: \mathcal{J}^{n} \rightarrow \mathcal{J}$ is given by thresholds $m_{p}, m_{q} \in\{0, \ldots, n+1\}$ and an 'exception domain' $\mathcal{E} \subseteq \mathcal{J}^{n}$, and is defined as follows for all voting profiles $\mathbf{v} \in \mathcal{J}$ : if $\mathbf{v} \notin \mathcal{E}$ then $f(\mathbf{v})$ contains any proposition $r$ in $\{p, q\}$ if and only if $n_{r}^{\mathbf{v}} \geq m_{r}$, while if $\mathbf{v} \in \mathcal{E}$ then $f(\mathbf{v})=p q$; or equivalently, $f(\mathbf{v})$ contains any $r$ in $\{p, q\}$ if and only if $\left[n_{r}^{\mathbf{v}} \geq m_{r}\right.$ or $\left.\left.\mathbf{v} \in \mathcal{E}\right]\right]^{14}$ Standard quota rules arise as special cases with an empty exception domain. In our characterization theorem, the exception domain is $\mathcal{E}=\left\{\mathbf{v}: \beta\left(n_{p}^{\mathbf{v}}, n_{q}^{\mathbf{v}}\right)>1 / 2\right\}$, so that

$$
f(\mathbf{v}) \text { contains } r \Leftrightarrow\left[n_{r}^{\mathbf{v}} \geq m_{r} \text { or } \beta\left(n_{p}^{\mathbf{v}}, n_{q}^{\mathbf{v}}\right)>1 / 2\right] \text {, for all } r \in\{p, q\} \text { and } \mathbf{v} \in \mathcal{J} \text {. }
$$

Theorem 5 Assume consequentialist preferences of type 2. A voting rule $f$ makes informative voting efficient and is monotonic and anonymous if and only if $f$ is the quota rule with exception (8) for some thresholds $m_{p}, m_{q}$ such that $\beta\left(m_{p}, l_{q}\right), \beta\left(l_{p}, m_{q}\right)>$ $1 / 2$.
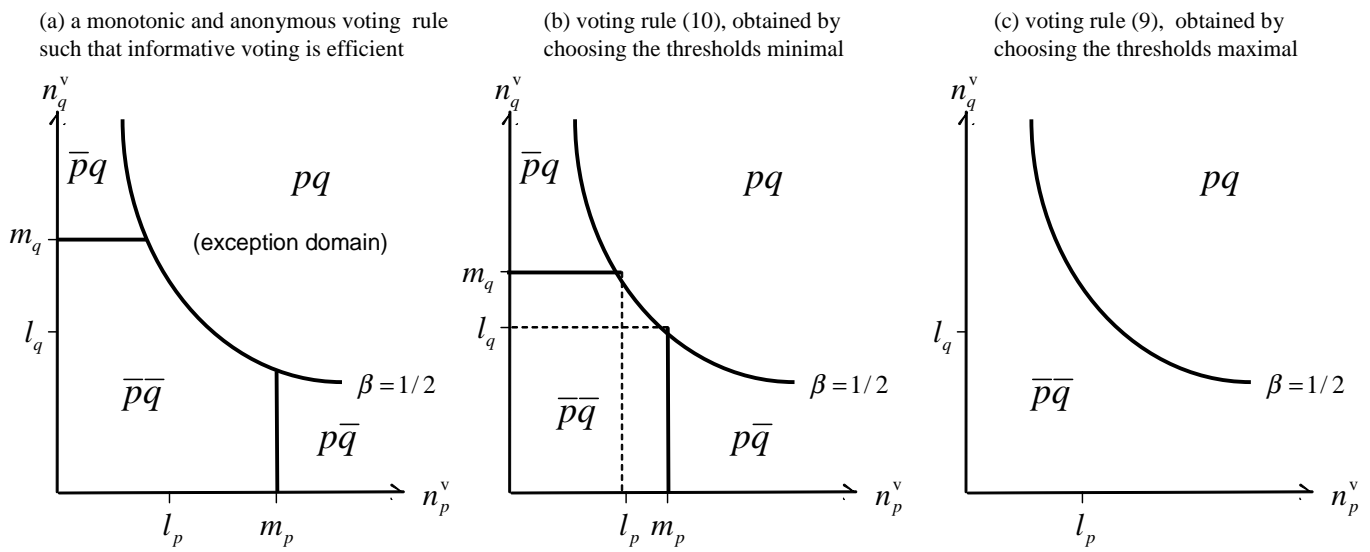

Figure 2: Illustration of Theorem 5: the decision as a function of the number of votes for $p$ and $q$

Figure 2 shows three voting rules of the kind given in Theorem 5, which differ in the choice of the thresholds $m_{p}$ and $m_{q}$. In Figure 2a, the thresholds are chosen in a 'non-extreme' way. In Figure 2c, the thresholds are maximal, i.e., $m_{p}=m_{q}=n+1$, so that the voting rule takes a particularly simple form:

$$
f(\mathbf{v})= \begin{cases}p q & \text { if } \beta\left(n_{p}^{\mathbf{v}}, n_{q}^{\mathbf{v}}\right)>1 / 2 \\ \bar{p} \bar{q} & \text { if } \beta\left(n_{p}^{\mathbf{v}}, n_{q}^{\mathbf{v}}\right) \leq 1 / 2\end{cases}
$$

\footnotetext{
${ }^{14}$ The notion of a quota rules with exception could be generalized by allowing the exception decision to differ from $p q$. The exception decision is $p q$ for us due to the privileged status of $p q$ under consequentialist preferences of type 2 .
} 
for all voting profiles $\mathbf{v} \in \mathcal{J}^{n}$. In Figure $2 \mathrm{~b}$, the thresholds are minimal, so that the voting rule is given as follows:

$$
f(\mathbf{v})= \begin{cases}p q & \text { if } \beta\left(n_{p}^{\mathbf{v}}, n_{q}^{\mathbf{v}}\right)>1 / 2 \\ p \bar{q} & \text { if } \beta\left(n_{p}^{\mathbf{v}}, n_{q}^{\mathbf{v}}\right) \leq 1 / 2 \text { and } \beta\left(n_{p}^{\mathbf{v}}, l_{q}\right)>1 / 2 \\ \bar{p} q & \text { if } \beta\left(n_{p}^{\mathbf{v}}, n_{q}^{\mathbf{v}}\right) \leq 1 / 2 \text { and } \beta\left(l_{p}, n_{q}^{\mathbf{v}}\right)>1 / 2 \\ \bar{p} \bar{q} & \text { otherwise }\end{cases}
$$

The latter rule is special in that it reduces to the quota rule making informative voting efficient (defined in Theorem 4) whenever such a quota rule exists.

\section{When is informative voting sincere?}

While the previous section focuses on mechanism design, the present section does not depend on the voting rule (mechanism). We focus on a single voter and answer the question of when informative voting is sincere, that is, when the naive strategy of 'following the evidence' is worthwhile for a sincere voter. For each type of preference, we fully characterize the parameter combinations for which this is so. We begin with simple preferences.

Theorem 6 Under simple preferences, the informative voting strategy is sincere if and only if $\frac{a_{\bar{r}}}{1-a_{r}} \geq \frac{\pi_{r}}{1-\pi_{r}} \geq \frac{1-a_{\bar{r}}}{a_{r}}$ for each $r \in\{p, q\}$.

This result has an intuitive interpretation. We know that necessarily the upper bound $\frac{a_{\bar{r}}}{1-a_{r}}$ for $\frac{\pi_{r}}{1-\pi_{r}}$ exceeds 1 and the lower bound $\frac{1-a_{\bar{r}}}{a_{r}}$ is below 1 , since $a_{r}, a_{\bar{r}}>1 / 2$. For very high or very low values of the prior probabilities $\pi_{r}$, the ratio $\frac{\pi_{r}}{1-\pi_{r}}$ is far from 1 , so that one of the bounds is violated and informative voting is not sincere. This makes sense since if voters have 'strong' prior beliefs, then the evidence collected cannot overrule the prior beliefs: sincere votes cease to be sensitive to evidence, i.e., depart from informative votes. By contrast, for less strong prior beliefs, the inequalities are satisfied, so that informative voting is sincere, i.e., it is worth following the evidence as a sincere voter.

Another useful perspective on the result is obtained by focusing not on the parameters $\pi_{r}$ representing prior beliefs, but on the parameters $a_{r}$ and $a_{\bar{r}}$ representing 'strength of evidence'. The larger $a_{r}$ and $a_{\bar{r}}$ are (i.e., the 'stronger' private evidence for $r$ and $\bar{r}$ is), the greater the upper bound for $\frac{\pi_{r}}{1-\pi_{r}}$ is and the smaller the lower bound is, which makes it easier to meet both inequalities. In summary, sufficiently strong evidence and/or sufficiently weak prior beliefs imply that it is worth voting informatively ('following the evidence') as a sincere voter.

Surprisingly, the characterization remains the same as we move from simple preferences to consequentialist preferences of type 1 (though the proof is quite different):

Theorem 7 Under consequentialist preferences of type 1, the informative voting strategy is sincere if and only if $\frac{a_{\bar{r}}}{1-a_{r}} \geq \frac{\pi_{r}}{1-\pi_{r}} \geq \frac{1-a_{\bar{r}}}{a_{r}}$ for each $r \in\{p, q\}$.

One can interpret this result in a similar way as done for simple preferences. 
Finally, we turn to consequentialist preferences of type 2. Here, the characterization is based on the following three coefficients:

$$
\begin{aligned}
A & :=\frac{\pi_{p}}{1-\pi_{p}} \times \frac{a_{\bar{q}}}{1-a_{q}}+\frac{\pi_{q}}{1-\pi_{q}} \times \frac{1-a_{\bar{p}}}{a_{p}}+\frac{1-a_{\bar{p}}}{a_{p}} \times \frac{a_{\bar{q}}}{1-a_{q}} \\
B & :=\frac{\pi_{p}}{1-\pi_{p}} \times \frac{1-a_{\bar{q}}}{a_{q}}+\frac{\pi_{q}}{1-\pi_{q}} \times \frac{a_{\bar{p}}}{1-a_{p}}+\frac{a_{\bar{p}}}{1-a_{p}} \times \frac{1-a_{\bar{q}}}{a_{q}} \\
C & :=\frac{\pi_{p}}{1-\pi_{p}} \times \frac{1-a_{\bar{q}}}{a_{q}}+\frac{\pi_{q}}{1-\pi_{q}} \times \frac{1-a_{\bar{p}}}{a_{p}}+\frac{1-a_{\bar{p}}}{a_{p}} \times \frac{1-a_{\bar{q}}}{a_{q}} .
\end{aligned}
$$

Theorem 8 Under consequentialist preferences of type 2, the informative voting strategy is sincere if and only if $A, B \geq \frac{\pi_{p}}{1-\pi_{p}} \times \frac{\pi_{q}}{1-\pi_{q}} \geq C$.

Although the characterizing inequalities are more complicated than for the previous two kinds of preference, an interpretation in terms of strength of evidence is again possible. If the voter's evidence is sufficiently strong (i.e., if $a_{p}, a_{\bar{p}}, a_{q}, a_{\bar{q}}$ are sufficiently close 1 ), then $C$ is well below 1 and $A$ and $B$ are well above 1 , so that the inequalities are likely to hold; as a result, informative voting is sincere, i.e., it is worth following the evidence as a sincere voter.

\section{References}

Austen-Smith, D., Banks, J. (1996) Information aggregation, rationality, and the Condorcet jury theorem. The American Political Science Review 90: 34-45

Austen-Smith, D., Feddersen, T. (2006) Deliberation, preference uncertainty and voting rules. American Political Science Review 100(2): 209-217

Austen-Smith, D., Feddersen, T. (2005) Deliberation and Voting Rules, in AustenSmith, D. and J. Duggan (eds) Social Choice and Strategic Decisions: Essays in Honor of Jeffrey S. Banks, Berlin: Springer

Bovens, L., Rabinowicz, W. (2006) Democratic answers to complex questions: an epistemic perspective. Synthese 150(1): 131-153

Coughlan, P. (2000) In defense of unanimous jury verdicts: mistrials, communication and strategic voting. The American Political Science Review 94(2): 375-393

Dietrich, F. (2006) Judgment aggregation: (im)possibility theorems. Journal of Economic Theory 126(1): 286-298

Dietrich, F. (2007) A generalised model of judgment aggregation. Social Choice and Welfare 28(4): 529-565

Dietrich, F. (2010) The possibility of judgment aggregation on agendas with subjunctive implications. Journal of Economic Theory 145(2): 603-638

Dietrich, F., List, C. (2007a) Arrow's theorem in judgment aggregation. Social Choice and Welfare 29(1): 19-33

Dietrich, F., List, C. (2007b) Strategy-proof judgment aggregation. Economics and Philosophy 23: 269-300

Dietrich, F., List, C. (2008) Judgment aggregation without full rationality. Social Choice and Welfare 31: 15-39

Dietrich, F., Mongin, P. (2010) The premise-based approach to judgment aggregation. Journal of Economic Theory 145(2): 562-582 
Dokow, E., Holzman, R. (2010a) Aggregation of binary evaluations. Journal of Economic Theory 145(2): 495-511

Dokow, E., Holzman, R. (2010b) Aggregation of binary evaluations with abstentions. Journal of Economic Theory 145(2): 544-561

Duggan, J., Martinelli, C. (2001) A Bayesian model of voting in juries. Games and Economic Behavior 37(2): 259-294

Feddersen, T., Pesendorfer, W.(1997) Information aggregation and voting behaviour in elections. Econometrica 65(5): 1029-1058

Feddersen, T., Pesendorfer, W. (1998) Convicting the innocent: the inferiority of unanimous jury verdicts under strategic voting. The American Political Science Review 92(1): 23-15

Gerardi, D., (2000) Jury verdicts and preference diversity. American Political Science Review 94: 395-406

Goertz, J. M., Maniquet, F. (2011) On the informational efficiency of simple scoring rules. Journal of Economic Theory, forthcoming

Guilbaud, G. (1952) Les théories de l'intérêt général et le problème logique de l'agrégation. Économie Appliquée 5: 501-584

List, C. (2005) The probability of inconsistencies in complex collective decisions. Social Choice and Welfare 24(1): 3-32

List, C., Pettit, P. (2002) Aggregating sets of judgments: an impossibility result. Economics and Philosophy 18(1): 89-110

List, C., Pettit, P. (2011) Group Agency: The Possibility, Design and Status of Corporate Agents. Oxford University Press

List, C., Polak, B. (2010) Introduction to judgment aggregation. Journal of Economic Theory 145(2): 441-466

Nehring, K., Puppe, C. (2002) Strategy-proof social choice on single-peaked domains: possibility, impossibility and the space between. Working paper, University of California at Davis

Nehring, K., Puppe, C. (2007) The structure of strategy-proof social choice. Part I: General characterization and possibility results on median spaces. Journal of Economic Theory 135: 269-305

Nehring, K. Puppe, C. (2008) Consistent judgement aggregation: the truth-functional case. Social Choice and Welfare 31: 41-57

Nehring, K., Puppe, C. (2010) Abstract Arrovian aggregation. Journal of Economic Theory 145(2): 467-494

Wilson R (1975) On the theory of aggregation. Journal of Economic Theory 10: 89-99

\section{A Appendix: proofs}

We begin by some preliminary derivations (Section A.1), and then prove our results in a new order obtained by clustering the results according to the kind of preference (Sections A.2-5).

Conventions. Recall the notation ' $f_{r}$ ' introduced in fn. 4 and the notation ' $S$ ' for the random variable generating the state $s$ in $\mathcal{J}$ introduced in fn. 8. Double-negations 
cancel each other out, i.e., $\overline{\bar{p}}$ stands for $p$, and $\overline{\bar{q}}$ for $q$. We refer to the two technical assumptions made at the end of Section 2.3 as 'non-degeneracy' and 'no efficiency ties', respectively.

\section{A.1 Preliminary derivations}

The joint probability of a state-types vector $(s, \mathbf{t})=\left(s_{p} s_{q}, t_{1 p} t_{1 q}, \ldots, t_{n p} t_{n q}\right) \in \mathcal{J}^{n+1}$ is

$$
\operatorname{Pr}(s, \mathbf{t})=\operatorname{Pr}(s) \operatorname{Pr}(\mathbf{t} \mid s)=\operatorname{Pr}(s) \prod_{i} \operatorname{Pr}\left(t_{i} \mid s\right)=\operatorname{Pr}\left(s_{p}\right) \operatorname{Pr}\left(s_{q}\right) \prod_{i} \operatorname{Pr}\left(t_{i p} \mid s_{p}\right) \operatorname{Pr}\left(t_{i q} \mid s_{q}\right),
$$

where the last two equations follow from our independence assumptions. A voter's probability of a state $s=p_{s} q_{s} \in \mathcal{J}$ given his type $t=p_{t} q_{t} \in \mathcal{J}$ is given by $\operatorname{Pr}(s \mid t)=$ $\operatorname{Pr}\left(p_{s} \mid p_{t}\right) \operatorname{Pr}\left(q_{s} \mid q_{t}\right)$, which reduces to

$$
\begin{aligned}
& \operatorname{Pr}(s \mid t)=\frac{\pi_{p_{s}} a_{p_{s}}}{\pi_{p_{s}} a_{p_{s}}+\pi_{\overline{p_{s}}}\left(1-a_{\overline{p_{s}}}\right)} \times \frac{\pi_{q_{s}} a_{q_{s}}}{\pi_{q_{s}} a_{q_{s}}+\pi_{\overline{q_{s}}}\left(1-a_{\overline{q_{s}}}\right)} \text { if } p_{s}=p_{t}, q_{s}=q_{t} \\
& \operatorname{Pr}(s \mid t)=\frac{\pi_{p_{s}} a_{p_{s}}}{\pi_{p_{s}} a_{p_{s}}+\pi_{\overline{p_{s}}}\left(1-a_{\overline{p_{s}}}\right)} \times \frac{\pi_{q_{s}}\left(1-a_{q_{s}}\right)}{\pi_{q_{s}}\left(1-a_{q_{s}}\right)+\pi_{\overline{q_{s}}} a_{\overline{q_{s}}}} \text { if } p_{s}=p_{t}, q_{s} \neq q_{t} \\
& \operatorname{Pr}(s \mid t)=\frac{\pi_{p_{s}}\left(1-a_{p_{s}}\right)}{\pi_{p_{s}}\left(1-a_{p_{s}}\right)+\pi_{\overline{p_{s}}} a_{\overline{p_{s}}}} \times \frac{\pi_{q_{s}} a_{q_{s}}}{\pi_{q_{s}} a_{q_{s}}+\pi_{\overline{q_{s}}}\left(1-a_{\overline{q_{s}}}\right)} \text { if } p_{s} \neq p_{t}, q_{s}=q_{t} \\
& \operatorname{Pr}(s \mid t)=\frac{\pi_{p_{s}}\left(1-a_{p_{s}}\right)}{\pi_{p_{s}}\left(1-a_{p_{s}}\right)+\pi_{\overline{p_{s}}} a_{\overline{p_{s}}}} \times \frac{\pi_{q_{s}}\left(1-a_{q_{s}}\right)}{\pi_{q_{s}}\left(1-a_{q_{s}}\right)+\pi_{\overline{q_{s}}} a_{\overline{q_{s}}}} \text { if } p_{s} \neq p_{t}, q_{s} \neq q_{t}
\end{aligned}
$$

The probability of the four states in $\mathcal{J}$ conditional on the full information $\mathbf{t} \in \mathcal{J}^{n}$ is given as follows, where $k:=n_{p}^{\mathrm{t}}$ and $l:=n_{q}^{\mathrm{t}}$ :

$$
\begin{aligned}
& \operatorname{Pr}(p q \mid \mathbf{t})=\frac{\pi_{p} a_{p}^{k}\left(1-a_{p}\right)^{n-k} \pi_{q} a_{q}^{l}\left(1-a_{q}\right)^{n-l}}{\operatorname{Pr}(\mathbf{t})} \\
& \operatorname{Pr}(p \bar{q} \mid \mathbf{t})=\frac{\pi_{p} a_{p}^{k}\left(1-a_{p}\right)^{n-k} \pi_{\bar{q}}\left(1-a_{\bar{q}}\right)^{l} a_{\bar{q}}^{n-l}}{\operatorname{Pr}(\mathbf{t})} \\
& \operatorname{Pr}(\bar{p} q \mid \mathbf{t})=\frac{\pi_{\bar{p}}\left(1-a_{\bar{p}}\right)^{k} a_{\bar{p}}^{n-k} \pi_{q} a_{q}^{l}\left(1-a_{q}\right)^{n-l}}{\operatorname{Pr}(\mathbf{t})} \\
& \operatorname{Pr}(\bar{p} \bar{q} \mid \mathbf{t})=\frac{\pi_{\bar{p}}\left(1-a_{\bar{p}}\right)^{k} a_{\bar{p}}^{n-k} \pi_{\bar{q}}\left(1-a_{\bar{q}}\right)^{l} a_{\bar{q}}^{n-l}}{\operatorname{Pr}(\mathbf{t})} .
\end{aligned}
$$

\section{A.2 General preferences}

Proof of Theorem 1. (a) We write $T_{i}\left(=T_{i p} T_{i q}\right)$ for the random variable generating voter $i$ 's type in $\mathcal{J}$, and $\mathbf{T}=\left(T_{1}, \ldots, T_{n}\right)$ for the random type profile. Consider any voting rule $f: \mathcal{J}^{n} \rightarrow \mathcal{J}$ and any efficient strategy profile $\boldsymbol{\sigma}=\left(\sigma_{1}, \ldots, \sigma_{n}\right)$. To show that $\boldsymbol{\sigma}$ is rational, consider any voter $i$ and type $t_{i} \in \mathcal{J}$. We have to show that $i$ 's vote $\sigma_{i}\left(t_{i}\right)$ maximizes the expected utility conditional on $i$ 's type, i.e., that

$$
E\left(u\left(f\left(\sigma_{i}\left(t_{i}\right), \boldsymbol{\sigma}_{-i}\left(\mathbf{T}_{-i}\right)\right), S\right) \mid t_{i}\right) \geq E\left(u\left(f\left(v_{i}, \boldsymbol{\sigma}_{-i}\left(\mathbf{T}_{-i}\right)\right), S\right) \mid t_{i}\right) \text { for all } v_{i} \in \mathcal{J},
$$


where $\left(\sigma_{i}\left(t_{i}\right), \boldsymbol{\sigma}_{-i}\left(\mathbf{T}_{-i}\right)\right)$ and $\left(v_{i}, \boldsymbol{\sigma}_{-i}\left(\mathbf{T}_{-i}\right)\right)$ of course denote the voting profiles in which $i$ votes $v_{i}$ resp. $\sigma_{i}\left(t_{i}\right)$ and each $j \neq i$ votes $\sigma_{j}\left(T_{j}\right)$. To show this, note that for all $v_{i} \in \mathcal{J}$,

$$
\begin{aligned}
E\left(u\left(f\left(v_{i}, \boldsymbol{\sigma}_{-i}\left(\mathbf{T}_{-i}\right)\right), S\right) \mid t_{i}\right) & =\sum_{\mathbf{t}_{-i} \in \mathcal{J}^{n-1}} \operatorname{Pr}\left(\mathbf{t}_{-i} \mid t_{i}\right) E\left(u\left(f\left(v_{i}, \boldsymbol{\sigma}_{-i}\left(\mathbf{t}_{-i}\right)\right), S\right) \mid t_{i}, \mathbf{t}_{-i}\right) \\
& \leq \sum_{\mathbf{t}_{-i} \in \mathcal{J}^{n-1}} \operatorname{Pr}\left(\mathbf{t}_{-i} \mid t_{i}\right) E\left(u\left(f\left(\sigma_{i}\left(t_{i}\right), \boldsymbol{\sigma}_{-i}\left(\mathbf{t}_{-i}\right)\right), S\right) \mid t_{i}, \mathbf{t}_{-i}\right) \\
& =E\left(u\left(f\left(\sigma_{i}\left(t_{i}\right), \boldsymbol{\sigma}_{-i}\left(\mathbf{T}_{-i}\right)\right), S\right) \mid t_{i}\right),
\end{aligned}
$$

where the inequality holds because the strategy profile $\left(\sigma_{i}, \boldsymbol{\sigma}_{-i}\right)=\boldsymbol{\sigma}$ is efficient for the type profile $\left(t_{i}, \mathbf{t}_{-i}\right)=\mathbf{t}$.

(b) Since by (15)-(18) the conditional distribution of the state given full information $\mathbf{t} \in \mathcal{J}^{n}$ depends on $\mathbf{t}$ only via the numbers $n_{p}^{\mathbf{t}}$ and $n_{q}^{\mathbf{t}}$, so does the conditional expected utility of each decision, and hence, the set of efficient decisions. For each $(k, l) \in\{0,1, \ldots, n\}^{2}$, let $F(k, l) \in \mathcal{J}$ be a decision that is efficient for some (hence, every) $\mathbf{t} \in \mathcal{J}^{n}$ for which $n_{p}^{\mathbf{t}}=k$ and $n_{q}^{\mathbf{t}}=l$. The voting rule $f$ defined by $\mathbf{v} \mapsto f(\mathbf{v})=F\left(n_{p}^{\mathbf{v}}, n_{q}^{\mathbf{v}}\right)$ is clearly anonymous and renders informative voting efficient.

\section{A.3 Simple preferences}

We begin by two lemmas.

Lemma 1 Assume simple preferences. The expected utility of a decision $d \in \mathcal{J}$ is

$$
E(u(d, S))=\operatorname{Pr}(S=d),
$$

and the conditional expected utility of d given a type or a type profile is given by the analogous expression with a conditional probability instead of an unconditional one.

Proof. The claim follows immediately from the definition of the utility function.

The next lemma invokes the coefficients $k_{p}$ and $k_{q}$ defined in (3) and (4).

Lemma 2 Assume simple preferences. For all type profiles $\mathbf{t} \in \mathcal{J}^{n}$, all $r \in\{p, q\}$, and all decisions $d, d^{\prime} \in \mathcal{J}$ such that $d$ but not $d^{\prime}$ contains $r$, and $d$ and $d^{\prime}$ share the other proposition,

$$
E(u(d, S) \mid \mathbf{t})>E\left(u\left(d^{\prime}, S\right) \mid \mathbf{t}\right) \Leftrightarrow n_{r}^{\mathbf{t}} \geq k_{r} .
$$

Proof. Let $\mathbf{t} \in \mathcal{J}^{n}$. We first prove the equivalence for $r=p, d=p q$ and $d^{\prime}=\bar{p} q$. By the definition of $k_{p}$, the inequality $n_{p}^{\mathrm{t}} \geq k_{p}$ is equivalent to

$$
\frac{\pi_{p}}{1-\pi_{p}}>\left(\frac{1-a_{\bar{p}}}{a_{p}}\right)^{n_{p}^{\mathbf{t}}}\left(\frac{a_{\bar{p}}}{1-a_{p}}\right)^{n-n_{p}^{\mathbf{t}}},
$$

which by (15) and (17) is equivalent to $\operatorname{Pr}(p q \mid \mathbf{t})>\operatorname{Pr}(\bar{p} q \mid \mathbf{t})$, and hence by Lemma 1 to $E(u(p q, S) \mid \mathbf{t})>E(u(\bar{p} q, S) \mid \mathbf{t})$. Next, suppose $r=p, d=p \bar{q}$ and $d^{\prime}=\bar{p} \bar{q}$. Using 
(16) and (18), the inequality (19) is equivalent to $\operatorname{Pr}(p \bar{q} \mid \mathbf{t})>\operatorname{Pr}(\bar{p} \bar{q} \mid \mathbf{t})$, and hence, to $E(u(p \bar{q}, S) \mid \mathbf{t})>E(u(\bar{p} \bar{q}, S) \mid \mathbf{t})$. The proof for the remaining cases is analogous.

We are now in a position to prove the two theorems about simple preferences.

Proof of Theorem 2. Consider a rule $f: \mathcal{J}^{n} \rightarrow \mathcal{J}$.

A. First, assume $f$ is the quota rule with thresholds $k_{p}$ and $k_{q}$. Consider a given type profile $\mathbf{t} \in \mathcal{J}^{n}$. Supposing that voters vote informatively, the resulting voting profile is $\mathbf{v}=\mathbf{t}$. We have to show that the decision $d:=f(\mathbf{v})$ is efficient for $\mathbf{t}$, i.e., that $\left(^{*}\right) E(u(d, S) \mid \mathbf{t})>E\left(u\left(d^{\prime}, S\right) \mid \mathbf{t}\right)$ for all $d^{\prime} \in \mathcal{J} \backslash\{d\}$. (We use '>' rather than ' $\geq$ ' in $(*)$ because of our 'no efficiency ties' assumption.) The property $(*)$ follows from Lemma 2. For instance, if $d=p q$, then by definition of $f$ we have $n_{p}^{\mathbf{t}} \geq k_{p}$ and $n_{q}^{\mathbf{t}} \geq k_{q}$, so that Lemma 2 implies the inequality in $\left(^{*}\right)$ for $d^{\prime}=\bar{p} q$ and $d^{\prime}=p \bar{q}$

For instance, if $d=p q$, then by definition of $f$ we have $n_{p}^{\mathbf{t}} \geq k_{p}$ and $n_{q}^{\mathbf{t}} \geq k_{q}$, so that Lemma 2 implies that

$$
E(u(p q, S) \mid \mathbf{t})>E(u(\bar{p} q, S) \mid \mathbf{t}), E(u(p \bar{q}, S) \mid \mathbf{t})>E(u(\bar{p} \bar{q}, S) \mid \mathbf{t}),
$$

which in turn implies $(*)$; and if $d=\bar{p} \bar{q}$, then $n_{p}^{\mathbf{t}}<k_{p}$ and $n_{q}^{\mathbf{t}}<k_{q}$, so that Lemma 2 implies that

$$
E(u(\bar{p} \bar{q}, S) \mid \mathbf{t})>E(u(p \bar{q}, S) \mid \mathbf{t}), E(u(\bar{p} q, S) \mid \mathbf{t})>E(u(p q, S) \mid \mathbf{t}),
$$

which again implies $(*)$.

B. Conversely, suppose informative voting is efficient under $f$. We consider any $\mathbf{v} \in \mathcal{J}^{n}$ and $r \in\{p, q\}$, and must show that $(* *) f_{r}(\mathbf{v})=r \Leftrightarrow n_{r}^{\mathbf{v}} \geq k_{r}$. Consider the type profile $\mathbf{t}=\mathbf{v}$. Since informative voting is efficient, the decision $d=f(\mathbf{v})$ is efficient for $\mathbf{t}(=\mathbf{v})$, i.e., satisfies condition $(*)$ above. Lemma 2 and $(*)$ together imply $\left({ }^{* *}\right)$. For instance, if $f(\mathbf{v})=p q$, then $\left({ }^{* *}\right)$ holds because, firstly, $f_{r}(\mathbf{v})=r$, and secondly, $n_{r}^{\mathbf{v}} \geq k_{r}$ by $(*)$ and Lemma 2 .

Proof of Theorem 6. A. First, assume informative voting is sincere. Equivalently, for any given type $t \in \mathcal{J}, E(u(d, S) \mid t)$ is maximal at $d=t$, i.e., by Lemma $1\left(^{*}\right) \operatorname{Pr}(d \mid t)$ is maximal at $d=t$. Applying $\left(^{*}\right)$ to type $t=p q$, we have $\operatorname{Pr}(p q \mid t) \geq \operatorname{Pr}(\bar{p} q \mid t)$, which implies $\frac{\pi_{p}}{1-\pi_{p}} \geq \frac{1-a_{\bar{p}}}{a_{p}}$ by (11) and (13). Now applying $\left(^{*}\right)$ to type $t=\bar{p} \bar{q}$, we obtain $\operatorname{Pr}(\bar{p} \bar{q} \mid t) \geq \operatorname{Pr}(p \bar{q} \mid t)$, which by (11) and (13) implies $\frac{a_{\bar{p}}}{1-a_{p}} \geq \frac{\pi_{p}}{1-\pi_{p}}$. We have shown both inequalities relating to $p$. The two inequalities relating to $q$ can be proved analogously.

B. Now suppose $\frac{a_{\bar{r}}}{1-a_{r}} \geq \frac{\pi_{r}}{1-\pi_{r}} \geq \frac{1-a_{\bar{r}}}{a_{r}}$ for each $r \in\{p, q\}$. We consider any type $t \in \mathcal{J}$ and have to show that the decision $d=t$ has maximal expected utility given $t$, or equivalently, that $(*)$ holds.

We show $\left(^{*}\right)$ first in the case $t=p q$. Here, the inequality $\frac{\pi_{p}}{1-\pi_{p}} \geq \frac{1-a_{\bar{p}}}{a_{p}}$ implies $\operatorname{Pr}(p q \mid t) \geq \operatorname{Pr}(\bar{p} q \mid t)$ by (11) and (13), and it implies $\operatorname{Pr}(p \bar{q} \mid t) \geq \operatorname{Pr}(\bar{p} \bar{q} \mid t)$ by (12) and (14). Further, the inequality $\frac{\pi_{q}}{1-\pi_{q}} \geq \frac{1-a_{\bar{q}}}{a_{q}}$ implies $\operatorname{Pr}(p q \mid t) \geq \operatorname{Pr}(p \bar{q} \mid t)$ by (11) and (12). This shows $(*)$ for $t=p q$.

Now we show $\left(^{*}\right)$ for the case $t=p \bar{q}$. As $\frac{\pi_{p}}{1-\pi_{p}} \geq \frac{1-a_{\bar{p}}}{a_{p}}$, we here have $\operatorname{Pr}(p \bar{q} \mid t) \geq$ $\operatorname{Pr}(\bar{p} \bar{q} \mid t)$ by (11) and (13), and we have $\operatorname{Pr}(p q \mid t) \geq \operatorname{Pr}(\bar{p} q \mid t)$ by (12) and (14). As 
$\frac{a_{\bar{q}}}{1-a_{q}} \geq \frac{\pi_{q}}{1-\pi_{q}}$, we also have $\operatorname{Pr}(p \bar{q} \mid t) \geq \operatorname{Pr}(p q \mid t)$ by (11) and (12). This proves $\left(^{*}\right)$ for $t=p \bar{q}$.

By similar arguments, one shows $\left(^{*}\right)$ for $t=\bar{p} q$ and for $t=\bar{p} \bar{q}$.

\section{A.4 Consequentialist preferences: type 1}

We begin by two lemmas, which are the counterparts of Lemmas 1 and 2 for the current preferences.

Lemma 3 Assume consequentialist preferences of type 1. The expected utility of a decision $d \in \mathcal{J}$ is

$$
E(u(d, S))= \begin{cases}\operatorname{Pr}(p q)+\operatorname{Pr}(\bar{p} \bar{q}) & \text { if } d \in\{p q, \bar{p} \bar{q}\} \\ \operatorname{Pr}(p \bar{q})+\operatorname{Pr}(\bar{p} q) & \text { if } d \in\{p \bar{q}, \bar{p} q\}\end{cases}
$$

and the conditional expected utility of d given a type or a type profile is given by the analogous expression with conditional probabilities instead of unconditional ones.

Proof. The claim follows easily from the definition of the utility function.

Lemma 4 Assume consequentialist preferences of type 1 . For each type profile $\mathbf{t} \in$ $\mathcal{J}^{n}$ and decisions $d \in\{p q, \bar{p} \bar{q}\}$ and $d^{\prime} \in\{p \bar{q}, \bar{p} q\}$

$$
E(u(d, S) \mid \mathbf{t})>E\left(u\left(d^{\prime}, S\right) \mid \mathbf{t}\right) \Leftrightarrow\left[n_{r}^{\mathbf{t}} \geq k_{r} \text { for both or no } r \in\{p, q\}\right] .
$$

Proof. Consider any $\mathbf{t} \in \mathcal{J}^{n}, d \in\{p q, \bar{p} \bar{q}\}$ and $d^{\prime} \in\{p \bar{q}, \bar{p} q\}$. Define $g_{r}(k):=$ $\pi_{r} a_{r}^{k}\left(1-a_{r}\right)^{n-k}$ and $g_{\bar{r}}(k):=\left(1-\pi_{r}\right)\left(1-a_{\bar{r}}\right)^{k} a_{\bar{r}}^{n-k}$ for all $r \in\{p, q\}$ and $k \in \mathbb{R}$. For each $r \in\{p, q\}$, the definition of $k_{r}$ can now be rewritten as $k_{r}=\min \{k \in\{0,1, \ldots, n+$ $\left.1\}: g_{r}(k)>g_{\bar{r}}(k)\right\}$. So, $(*)$ for each $k \in\{0,1, \ldots, n+1\}, k \geq k_{r} \Leftrightarrow g_{r}(k)>g_{\bar{r}}(k)$. (Here, the implication ' $\Rightarrow$ ' uses that $g_{r}(k)\left[g_{\bar{r}}(k)\right]$ is strictly increasing [decreasing] in $k \in \mathbb{R}$.) Now,

$$
\begin{array}{ll} 
& E(u(d, S) \mid \mathbf{t})>E\left(u\left(d^{\prime}, S\right) \mid \mathbf{t}\right) \\
\Leftrightarrow & \operatorname{Pr}(p q \mid \mathbf{t})+\operatorname{Pr}(\bar{p} \bar{q} \mid \mathbf{t})>\operatorname{Pr}(p \bar{q} \mid \mathbf{t})+\operatorname{Pr}(\bar{p} q \mid \mathbf{t}) \text { by Lemma } 3 \\
\Leftrightarrow & g_{p}\left(n_{p}^{\mathbf{t}}\right) g_{q}\left(n_{q}^{\mathbf{t}}\right)+g_{\bar{p}}\left(n_{p}^{\mathbf{t}}\right) g_{\bar{q}}\left(n_{q}^{\mathbf{t}}\right)>g_{p}\left(n_{p}^{\mathbf{t}}\right) g_{\bar{q}}\left(n_{q}^{\mathbf{t}}\right)+g_{\bar{p}}\left(n_{p}^{\mathbf{t}}\right) g_{q}\left(n_{q}^{\mathbf{t}}\right) \text { by }(15)-(18) \\
\Leftrightarrow & {\left[g_{p}\left(n_{p}^{\mathbf{t}}\right)-g_{\bar{p}}\left(n_{p}^{\mathbf{t}}\right)\right]\left[g_{q}\left(n_{q}^{\mathbf{t}}\right)-g_{\bar{q}}\left(n_{q}^{\mathbf{t}}\right)\right]>0} \\
\Leftrightarrow & {\left[n_{r}^{\mathbf{t}} \geq k_{r} \text { for both or no } r \in\{p, q\}\right] \text { by }(*) .}
\end{array}
$$

We can now prove our two theorems about the present preferences.

Proof of Theorem 3. Consider a rule $f: \mathcal{J}^{n} \rightarrow \mathcal{J}$.

A. Assume $f$ is the quota rule with thresholds $k_{p}$ and $k_{q}$. Firstly, $f$ is monotonic. Secondly, to show that informative voting is efficient, consider a given type profile $\mathbf{t} \in \mathcal{J}^{n}$. Supposing informative voting, the resulting voting profile is then $\mathbf{v}:=\mathbf{t}$. We have to show that $d:=f(\mathbf{v})$ is efficient for $\mathbf{t}$, i.e., that for each $d^{\prime} \in \mathcal{J}$ with $\mathrm{Co}\left(d^{\prime}\right) \neq \mathrm{Co}(d)$ we have $\left(^{*}\right) E(u(d, S) \mid \mathbf{t}) \geq E\left(u\left(d^{\prime}, S\right) \mid \mathbf{t}\right)$. Consider any $d^{\prime} \in \mathcal{J}$ with $\operatorname{Co}\left(d^{\prime}\right) \neq \operatorname{Co}(d)$. If $d=p q$, then $n_{r}^{\mathrm{t}} \geq k_{r}$ for both $r \in\{p, q\}$, implying (*) by Lemma 
4. If $d=\bar{p} \bar{q}$, then $n_{r}^{\mathbf{t}} \geq k_{r}$ for no $r \in\{p, q\}$, again implying $\left(^{*}\right)$ by Lemma 4. Finally, if $d$ is $\bar{p} q$ or $p \bar{q}$, then $n_{r}^{\mathbf{t}} \geq k_{r}$ for exactly one $r \in\{p, q\}$, so that $\left(^{*}\right)$ holds once again by Lemma 4 .

B. Conversely, assume $f$ is monotonic and makes informative voting efficient. We consider any $\mathbf{v} \in \mathcal{J}^{n}$ and must show that $(* *) f_{r}(\mathbf{v})=r \Leftrightarrow n_{r}^{\mathbf{v}} \geq k_{r}$ for each $r \in\{p, q\}$. As one can show using our non-degeneracy assumption,

$$
k_{r} \notin\{0, n+1\} \text { for some } r \in\{p, q\}
$$

for instance, if $k_{r}$ were zero for each $r \in\{p, q\}$, then by Lemma 4 the decisions $\bar{p} q$ and $p \bar{q}$ would be inefficient for each type profile, violating non-degeneracy. We now prove $(* *)$ by distinguishing four cases.

Case 1: $n_{r}^{\mathbf{v}} \geq k_{r}$ for each $r \in\{p, q\}$. We must show that $f(\mathbf{v})=p q$. Since the decision $f(\mathbf{v})$ is efficient for the type profile $\mathbf{t}=\mathbf{v}$, by Lemma $4, f(\mathbf{v}) \in\{p q, \bar{p} \bar{q}\}$. Suppose for a contradiction $f(\mathbf{v})=\bar{p} \bar{q}$. By (20), $k_{r} \geq 1$ for some $r \in\{p, q\}$. Suppose $k_{p}>0$ (the case that $k_{q}>0$ being analogous). Let $\mathbf{v}^{\prime}$ be the voting profile obtained from $\mathbf{v}$ by replacing each occurring $p$ by $\bar{p}$. By monotonicity, the decision is $f\left(\mathbf{v}^{\prime}\right)=\bar{p} \bar{q}$. By Lemma 4 , for the type profile $\mathbf{t}^{\prime}=\mathbf{v}^{\prime}$ only $\bar{p} q$ and $p \bar{q}$ are efficient since $n_{p}^{\mathbf{t}^{\prime}}=0<k_{p}$ and $n_{q}^{\mathbf{t}^{\prime}}=n_{q}^{\mathbf{v}} \geq k_{q}$. So, the decision $f\left(\mathbf{v}^{\prime}\right)(=\bar{p} \bar{q})$ is inefficient, a contradiction since $f$ makes informative voting efficient.

Case 2: $n_{p}^{\mathbf{v}} \geq k_{p}$ and $n_{q}^{\mathbf{v}}<k_{q}$. We must show that $f(\mathbf{v})=p \bar{q}$. By Lemma 4 , $p \bar{q}$ and $\bar{p} q$ are both efficient for the type profile $\mathbf{t}=\mathbf{v}$. So, as informative voting is efficient, $f(\mathbf{v}) \in\{p \bar{q}, \bar{p} q\}$. Suppose for a contradiction $f(\mathbf{v})=\bar{p} q$. By $(20), k_{p}>0$ or $k_{q} \leq n$. First, if $k_{p}>0$, define $\mathbf{v}^{\prime}$ as in Case 1. By monotonicity, the decision is $f\left(\mathbf{v}^{\prime}\right)=\bar{p} q$, which is inefficient for the type profile $\mathbf{t}^{\prime}=\mathbf{v}^{\prime}$ by Lemma 4 as $n_{p}^{\mathbf{t}^{\prime}}=0<k_{p}$ and $n_{q}^{\mathbf{t}^{\prime}}=n_{q}^{\mathbf{v}}<k_{q}$, a contradiction. Second, if $k_{q} \leq n$, define $\mathbf{v}^{\prime}$ as the voting profile obtained from $\mathbf{v}$ by replacing each occurring $\bar{q}$ by $q$. By monotonicity, the decision is $f\left(\mathbf{v}^{\prime}\right)=\bar{p} q$, which is again inefficient for the type profile $\mathbf{t}^{\prime}=\mathbf{v}^{\prime}$ by Lemma 4 as $n_{p}^{\mathbf{t}^{\prime}}=n_{p}^{\mathbf{v}} \geq k_{p}$ and $n_{q}^{\mathbf{t}^{\prime}}=n \geq k_{q}$, a contradiction.

Case 3: $n_{p}^{\mathbf{v}}<k_{p}$ and $n_{q}^{\mathbf{v}} \geq k_{q}$. One can show that $f(\mathbf{v})=\bar{p} q$ like in Case 2 .

Case 4: $n_{r}^{\mathbf{v}}<k_{r}$ for each $r \in\{p, q\}$. We must show that $f(\mathbf{v})=\bar{p} \bar{q}$. By informative voting being efficient and by Lemma 4 applied to $\mathbf{t}=\mathbf{v}, f(\mathbf{v}) \in\{p q, \bar{p} \bar{q}\}$. Suppose for a contradiction that $f(\mathbf{v})=p q$. By (20), $k_{r} \leq n$ for some $r \in\{p, q\}$. We assume that $k_{p} \leq n$ (the proof being analogous if $k_{q} \leq n$ ). Let the voting profile $\mathbf{v}^{\prime} \in \mathcal{J}^{n}$ arise from $\mathbf{v}$ by replacing each occurring $\bar{p}$ by $p$. By monotonicity, $f\left(\mathbf{v}^{\prime}\right)=p q$. This outcome is inefficient for the type profile $\mathbf{t}^{\prime}=\mathbf{v}^{\prime}$ by Lemma 4 and $n_{p}^{\mathbf{t}^{\prime}}=n \geq k_{p}$ and $n_{q}^{\mathbf{t}^{\prime}}=n_{q}^{\mathbf{v}}<k_{q}$.

Proof of Theorem 7. A. First, let informative voting be sincere. Equivalently, for any type $t \in \mathcal{J},\left(^{*}\right) E(u(d, S) \mid t)$ is maximal at $d=t$. Using $\left(^{*}\right)$ with $t=p q$, we have $E(u(p q, S) \mid t) \geq E(u(\bar{p} q, S) \mid t)$, which by Lemma 3 is equivalent to $\operatorname{Pr}(p q \mid t)+\operatorname{Pr}(\bar{p} \bar{q} \mid t) \geq$ $\operatorname{Pr}(p \bar{q} \mid t)+\operatorname{Pr}(\bar{p} q \mid t)$. Using (11)-(14), the latter is equivalent to

$$
\frac{\pi_{p}}{1-\pi_{p}} \times \frac{\pi_{q}}{1-\pi_{q}}+\frac{1-a_{\bar{p}}}{a_{p}} \times \frac{1-a_{\bar{q}}}{a_{q}} \geq \frac{\pi_{p}}{1-\pi_{p}} \times \frac{1-a_{\bar{q}}}{a_{q}}+\frac{\pi_{q}}{1-\pi_{q}} \times \frac{1-a_{\bar{p}}}{a_{p}},
$$


which can be rearranged as

$$
\left(\frac{\pi_{p}}{1-\pi_{p}}-\frac{1-a_{\bar{p}}}{a_{p}}\right)\left(\frac{\pi_{q}}{1-\pi_{q}}-\frac{1-a_{\bar{q}}}{a_{q}}\right) \geq 0
$$

Analogously, using $\left(^{*}\right)$ three more times, with $t=p \bar{q}$, then $t=\bar{p} q$ and finally $t=\bar{p} \bar{q}$, we obtain

$$
\begin{aligned}
& \left(\frac{\pi_{p}}{1-\pi_{p}}-\frac{1-a_{\bar{p}}}{a_{p}}\right)\left(\frac{1-\pi_{q}}{\pi_{q}}-\frac{1-a_{q}}{a_{\bar{q}}}\right) \geq 0 \\
& \left(\frac{\pi_{p}}{1-\pi_{p}}-\frac{a_{\bar{p}}}{1-a_{p}}\right)\left(\frac{1-\pi_{q}}{\pi_{q}}-\frac{a_{q}}{1-a_{\bar{q}}}\right) \geq 0 \\
& \left(\frac{\pi_{p}}{1-\pi_{p}}-\frac{a_{\bar{p}}}{1-a_{p}}\right)\left(\frac{\pi_{q}}{1-\pi_{q}}-\frac{a_{\bar{q}}}{1-a_{q}}\right) \geq 0 .
\end{aligned}
$$

Firstly, (i) $\frac{\pi_{q}}{1-\pi_{q}} \geq \frac{1-a_{\bar{q}}}{a_{q}}$, since otherwise by (21) we would get $\frac{\pi_{p}}{1-\pi_{p}} \leq \frac{1-a_{\bar{p}}}{a_{p}}(<1)$, whereas by (23) we get $\frac{\pi_{p}}{1-\pi_{p}} \geq \frac{a_{\bar{p}}}{1-a_{p}}\left(>1\right.$ ), a contradiction. Secondly, (ii) $\frac{\pi_{p}}{1-\pi_{p}} \geq$ $\frac{1-a_{\bar{p}}}{a_{p}}$, because if (i) holds with a strict inequality, then (ii) follows from (21), whereas if (i) holds with equality, then $\frac{\pi_{q}}{1-\pi_{q}}<1<\frac{a_{\bar{q}}}{1-a_{q}}$, which together with (22) implies (ii). We finally show that (iii) $\frac{\pi_{p}}{1-\pi_{p}} \leq \frac{a_{\bar{p}}}{1-a_{p}}$ and (iv) $\frac{\pi_{q}}{1-\pi_{q}} \leq \frac{a_{\bar{q}}}{1-a_{q}}$. First, suppose (ii) holds with equality. Then $\frac{\pi_{p}}{1-\pi_{p}}<1<\frac{a_{\bar{p}}}{1-a_{p}}$, which implies (iii), and with (24) also implies (iv). Second, suppose (ii) holds with a strict inequality. Then with (22) we get (iv). If (iv) holds with a strict inequality, then we get (iii) by (24), while if (iv) holds with equality, then $\frac{1-\pi_{q}}{\pi_{q}}=\frac{1-a_{q}}{a_{\bar{q}}}<1<\frac{a_{q}}{1-a_{\bar{q}}}$, which by (23) implies (iii).

B. Conversely, assume $\frac{a_{\bar{r}}}{1-a_{r}} \geq \frac{\pi_{r}}{1-\pi_{r}} \geq \frac{1-a_{\bar{r}}}{a_{r}}$ for each $r \in\{p, q\}$. We have to show that informative voting is sincere, i.e., that $(*)$ holds for each type $t \in \mathcal{J}$. As one can check, the inequalities (21)-(24) all hold. These inequalities imply that $\left(^{*}\right)$ holds for each type $t \in \mathcal{J}$. For instance, as shown in part A, (21) reduces to $E(u(p q, S) \mid t) \geq E(u(\bar{p} q, S) \mid t)$ for $t=p q$.

\section{A.5 Consequentialist preferences: type 2}

We begin by a simple lemma, the counterpart of Lemmas 1 and 3 .

Lemma 5 Assume consequentialist preferences of type 2. The expected utility of a decision $d \in \mathcal{J}$ is

$$
E(u(d, S))= \begin{cases}\operatorname{Pr}(p q) & \text { if } d=p q \\ 1-\operatorname{Pr}(p q) & \text { if } d \neq p q\end{cases}
$$

and the conditional expected utility of d given a type or a type profile is given by the analogous expression with conditional probabilities instead of unconditional ones.

Proof. The claim follows from the specification of the utility function.

We now prove our results about the current preferences. Some proofs implicitly extend $\beta(k, l)$ to values of $k . l$ not in $\{0, \ldots, n\}$, using the expression $(5)$. 
Proof of proposition 1. The claim can easily be shown by elaborating the informal argument given in the text.

Proof of theorem 4. A. First, suppose $f: \mathcal{J}^{n} \rightarrow \mathcal{J}$ is a quota rule with thresholds $m_{p}$ and $m_{q}$ making informative voting efficient. The following claims must be shown.

Claim 1: $m_{p}=l_{p}$ and $m_{q}=l_{q}$.

Consider a type profile $\mathbf{t} \in \mathcal{J}^{n}$ for which $n_{p}^{\mathbf{t}}=n$ and $n_{q}^{\mathbf{t}}=l_{q}$. Assuming informative voting, the resulting voting profile is $\mathbf{v}=\mathbf{t}$. By definition of $l_{q}, \beta\left(n, l_{q}\right)>1 / 2$. So $f(\mathbf{v})=p q$ by Proposition 1 . Thus, $l_{q} \geq m_{q}$ by definition of $f$. One analogously shows that $l_{p} \geq m_{p}$. To show the converse inequalities, consider a voting profile $\mathbf{v} \in \mathcal{J}^{n}$ for which $n_{p}^{\mathbf{v}}=m_{p}$ and $n_{q}^{\mathbf{v}}=n\left(\geq m_{q}\right)$. The resulting decision is $f(\mathbf{v})=p q$ by definition of $f$. So, by Proposition $1, \beta\left(n_{p}^{\mathbf{v}}, n_{q}^{\mathbf{v}}\right)=\beta\left(m_{p}, n\right)>1 / 2$. Hence, $m_{p} \geq l_{p}$ by definition of $l_{p}$. Analogously, one shows that $m_{q} \geq l_{q}$.

Claim 2: $\beta\left(l_{p}, l_{q}\right)>1 / 2$.

For any voting profile $\mathbf{v} \in \mathcal{J}^{n}$ for which $n_{p}^{\mathbf{v}}=l_{p}\left(=m_{p}\right)$ and $n_{q}^{\mathbf{v}}=l_{q}\left(=m_{q}\right)$, we have $f(\mathbf{v})=p q$ by definition of $f$, so that by Proposition $1 \beta\left(n_{p}^{\mathbf{v}}, n_{q}^{\mathbf{v}}\right)>1 / 2$, i.e., $\beta\left(l_{p}, l_{q}\right)>1 / 2$.

B. Conversely, assume $\beta\left(l_{p}, l_{q}\right)>1 / 2$. We show that the quota rule $f$ with thresholds $l_{p}$ and $l_{q}$ makes informative voting efficient. We first prove that for all $k, l \in\{0, \ldots, n\}$,

$$
\beta(k, l)>1 / 2 \Leftrightarrow\left[k \geq l_{p} \text { and } l \geq l_{q}\right] .
$$

Let $k, l \in\{0, \ldots, n\}$. If $k \geq l_{p}$ and $l \geq l_{q}$, then $\beta(k, l) \geq \beta\left(l_{p}, l_{q}\right)>1 / 2$, where the first inequality holds because $\beta$ is increasing in each argument. If $k<l_{p}$, then $\beta(k, l) \leq \beta(k, n) \leq 1 / 2$, where the last inequality holds by definition of $l_{p}(>k)$. Analogously, if $l \leq l_{q}$, then $\beta(k, l) \leq 1 / 2$.

Now consider any type profile $\mathbf{t} \in \mathcal{J}^{n}$. Assuming informative voting, the resulting voting profile is $\mathbf{v}=\mathbf{t}$. We have to show that the decision $f(\mathbf{v})$ is efficient for $\mathbf{t}(=\mathbf{v})$. First, if $n_{p}^{\mathbf{t}} \geq l_{p}$ and $n_{q}^{\mathbf{t}} \geq l_{q}$, the decision is $f(\mathbf{v})=p q$, which is efficient by Proposition 1 since $\beta\left(n_{p}^{\mathbf{t}}, n_{q}^{\mathbf{t}}\right)>1 / 2$ by $(25)$. Second, if $n_{p}^{\mathbf{t}}<l_{p}$ or $n_{q}^{\mathbf{t}}<l_{q}$, the resulting decision $f(\mathbf{v})$ is in $\{\bar{p} q, p \bar{q}, \bar{p} \bar{q}\}$, which is efficient by Proposition 1 since $\beta\left(n_{p}^{\mathbf{t}}, n_{q}^{\mathbf{t}}\right) \leq 1 / 2$ by $(25)$.

Proof of Theorem 5 . Consider a rule $f: \mathcal{J}^{n} \rightarrow \mathcal{J}$. We repeatedly draw on the fact that $\left(^{*}\right) \beta(k, l)$ is strictly increasing in each argument.

A. First, assume $f$ is defined by (8) for thresholds $m_{p}$ and $m_{q}$ satisfying $\beta\left(m_{p}, l_{q}\right)$, $\beta\left(l_{p}, m_{q}\right)>1 / 2$. Clearly, $f$ is anonymous. To show that informative voting is efficient, it suffices by Proposition 1 to prove that for all $\mathbf{v} \in \mathcal{J}^{n}$,

$$
f(\mathbf{v})=p q \Leftrightarrow \beta\left(n_{p}^{\mathbf{v}}, n_{q}^{\mathbf{v}}\right)>1 / 2 .
$$

If $\beta\left(n_{p}^{\mathbf{v}}, n_{q}^{\mathbf{v}}\right)>1 / 2$, then clearly $f(\mathbf{v})=p q$ by (8). Conversely, assume $f(\mathbf{v})=p q$. Then, by definition of $f$, either $n_{r}^{\mathbf{v}} \geq m_{r}$ for each $r \in\{p, q\}$, or $\beta\left(n_{p}^{\mathbf{v}}, n_{q}^{\mathbf{v}}\right)>1 / 2$. In the second case, we are done. Now assume the first case. Since $\beta\left(m_{p}, l_{q}\right)>1 / 2$, we have $\beta\left(m_{p}, n\right)>1 / 2$ by $(*)$, whence $m_{p} \geq l_{p}$ by definition of $l_{p}$. Using $\left(^{*}\right)$ and that $n_{p}^{\mathbf{v}} \geq m_{p} \geq l_{p}$ and $n_{q}^{\mathbf{v}} \geq m_{q}$, we have $\beta\left(n_{p}^{\mathbf{v}}, n_{q}^{\mathbf{v}}\right) \geq \beta\left(l_{p}, m_{q}\right)$. Moreover, 
$\beta\left(l_{p}, m_{q}\right)>1 / 2$ by definition of $m_{q}$. So, $\beta\left(n_{p}^{\mathbf{v}}, n_{q}^{\mathbf{v}}\right)>1 / 2$, which completes the proof of $(26)$.

It remains to show monotonicity of $f$. Take two voting profiles $\mathbf{v}, \mathbf{v}^{\prime} \in \mathcal{J}^{n}$ such that for all $r \in f(\mathbf{v})$, the voters who vote for $r$ in $\mathbf{v}$ also vote for $r$ in $\mathbf{v}^{\prime}$.

Case 1: $f(\mathbf{v})=p q$. Then, $\beta\left(n_{p}^{\mathbf{v}}, n_{q}^{\mathbf{v}}\right)>1 / 2$ by (26). Also, $n_{p}^{\mathbf{v}^{\prime}} \geq n_{p}^{\mathbf{v}}$ and $n_{q}^{\mathbf{v}^{\prime}} \geq n_{q}^{\mathbf{v}}$, so that $\beta\left(n_{p}^{\mathbf{v}^{\prime}}, n_{q}^{\mathbf{v}^{\prime}}\right) \geq \beta\left(n_{p}^{\mathbf{v}}, n_{q}^{\mathbf{v}}\right)$ by $\left({ }^{*}\right)$. It follows that $\beta\left(n_{p}^{\mathbf{v}^{\prime}}, n_{q}^{\mathbf{v}^{\prime}}\right)>1 / 2$, so that $f\left(\mathbf{v}^{\prime}\right)=p q$ by $(26)$.

Case 2: $f(\mathbf{v})=p \bar{q}$. We have to show that $f\left(\mathbf{v}^{\prime}\right)=p \bar{q}$, i.e., that

$$
n_{p}^{\mathbf{v}^{\prime}} \geq m_{p}, n_{q}^{\mathbf{v}^{\prime}}<m_{q}, \text { and } \beta\left(n_{p}^{\mathbf{v}^{\prime}}, n_{q}^{\mathbf{v}^{\prime}}\right) \leq 1 / 2 .
$$

Since $f(\mathbf{v})=p \bar{q}$, the definition of $f$ implies $n_{p}^{\mathbf{v}} \geq m_{p}$ and $n_{q}^{\mathbf{v}}<m_{q}$, and the definition of $\mathbf{v}^{\prime}$ implies $n_{p}^{\mathbf{v}^{\prime}} \geq n_{p}^{\mathbf{v}}$ and $n_{q}^{\mathbf{v}^{\prime}} \leq n_{q}^{\mathbf{v}}$; hence, the first two inequalities in (27) hold. As $\beta\left(m_{p}, l_{q}\right)>1 / 2$ and $n_{p}^{\mathbf{v}} \geq m_{p}$, we have $\beta\left(n_{p}^{\mathbf{v}}, l_{q}\right)>1 / 2$ by $\left(^{*}\right)$. Also, since $f(\mathbf{v})=p \bar{q}$, we have $\beta\left(n_{p}^{\mathbf{v}}, n_{q}^{\mathbf{v}}\right) \leq 1 / 2$ by $(26)$. Hence, $\beta\left(n_{p}^{\mathbf{v}}, n_{q}^{\mathbf{v}}\right)<\beta\left(n_{p}^{\mathbf{v}}, l_{q}\right)$. So, $n_{q}^{\mathbf{v}}<l_{q}$ by $\left({ }^{*}\right)$, whence $n_{q}^{\mathbf{v}^{\prime}}<l_{q}$ as $n_{q}^{\mathbf{v}^{\prime}} \leq n_{q}^{\mathbf{v}}$. Thus, by definition of $l_{q}, \beta\left(n, n_{q}^{\mathbf{v}^{\prime}}\right) \leq 1 / 2$, so that $\beta\left(n_{p}^{\mathbf{v}^{\prime}}, n_{q}^{\mathbf{v}^{\prime}}\right) \leq 1 / 2$ by $(*)$, proving $(27)$.

Case 3: $f(\mathbf{v})=\bar{p} q$. One can show that $f\left(\mathbf{v}^{\prime}\right)=\bar{p} q$ analogously to Case 2 .

Case 4: $f(\mathbf{v})=\bar{p} \bar{q}$. Then, $n_{p}^{\mathbf{v}}<m_{p}, n_{q}^{\mathbf{v}}<m_{q}$, and $\beta\left(n_{p}^{\mathbf{v}}, n_{q}^{\mathbf{v}}\right) \leq 1 / 2$. We have to show that $f\left(\mathbf{v}^{\prime}\right)=\bar{p} \bar{q}$, i.e., that these three inequalities still hold if $\mathbf{v}$ is replaced by $\mathbf{v}^{\prime}$. This follows from the fact that $n_{p}^{\mathbf{v}^{\prime}} \leq n_{p}^{\mathbf{v}}$ and $n_{q}^{\mathbf{v}^{\prime}} \leq n_{q}^{\mathbf{v}}$ (by definition of $\mathbf{v}^{\prime}$ ) and from $(*)$.

B. Conversely, let $f$ be monotonic and anonymous, and make informative voting efficient. For each $r \in\{p, q\}$, define

$$
m_{r}:=\min \left\{n_{r}^{\mathbf{v}}: \mathbf{v} \in \mathcal{J}^{n} \text { such that } f_{r}(\mathbf{v})=r \text { and } \beta\left(n_{p}^{\mathbf{v}}, n_{q}^{\mathbf{v}}\right) \leq 1 / 2\right\},
$$

where this minimum is interpreted as $n+1$ if it is taken over an empty set. We prove that $f$ has the required form with respect to the so-defined thresholds $m_{p}$ and $m_{q}$. The proof proceeds in several steps and is completed by Claims 5 and 6 below.

Claim 1: For all $\mathbf{v} \in \mathcal{J}^{n}$, if $n_{p}^{\mathbf{v}} \geq l_{p}, n_{q}^{\mathbf{v}} \geq l_{q}$ and $\beta\left(n_{p}^{\mathbf{v}}, n_{q}^{\mathbf{v}}\right) \leq 1 / 2$, then $f(\mathbf{v})=\bar{p} \bar{q}$.

Let $\mathbf{v} \in \mathcal{J}^{n}$ satisfy the antecedent condition. First assume $f(\mathbf{v})=p \bar{q}$ for a contradiction. Let $\mathbf{v}^{\prime}$ be the voting profile obtained from $\mathbf{v}$ by replacing each $\bar{p}$ by $p$. By monotonicity, $f\left(\mathbf{v}^{\prime}\right)=p \bar{q}$. However, Proposition 1 implies that $f\left(\mathbf{v}^{\prime}\right)=p q$, since $\beta\left(n_{p}^{\mathbf{v}^{\prime}}, n_{q}^{\mathbf{v}^{\prime}}\right)=\beta\left(n, n_{q}^{\mathbf{v}}\right) \geq \beta\left(n, l_{q}\right)>1 / 2$ (where the first inequality holds by $n_{q}^{\mathbf{v}} \geq l_{q}$, and the second by definition of $\left.l_{q}\right)$. This contradiction proves that $f(\mathbf{v}) \neq p \bar{q}$. One similarly proves that $f(\mathbf{v}) \neq \bar{p} q$. So, as $f(\mathbf{v}) \in\{p \bar{q}, \bar{p} q, \bar{p} \bar{q}\}$ by Proposition 1 , we have $f(\mathbf{v})=\bar{p} \bar{q}$, proving the claim.

Claim 2: For all $\mathbf{v} \in \mathcal{J}^{n}$, if $n_{p}^{\mathbf{v}} \leq l_{p}, n_{q}^{\mathbf{v}} \leq l_{q}$ and $\beta\left(l_{p}, l_{q}\right) \leq 1 / 2$, then $f(\mathbf{v})=\bar{p} \bar{q}$.

Consider any $\mathbf{v} \in \mathcal{J}^{n}$ satisfying the antecedent condition. Let $\mathbf{w} \in \mathcal{J}^{n}$ arise from $\mathbf{v}$ by replacing $l_{p}-n_{p}^{\mathbf{v}}$ occurrences of $\bar{p}$ by $p, l_{q}-n_{q}^{\mathbf{v}}$ occurrences of $\bar{q}$ by $q$. Note that $n_{p}^{\mathbf{w}}=l_{p}$ and $n_{q}^{\mathbf{w}}=l_{q}$, whence by Claim $1 f(\mathbf{w})=\bar{p} \bar{q}$. By monotonicity, it follows that $f(\mathbf{v})=\bar{p} \bar{q}$.

Claim 3: $m_{p} \geq l_{p}$ and $m_{q} \geq l_{q}$.

Suppose for a contradiction $m_{p}<l_{p}$. By definition of $m_{p}$, there is a $\mathbf{v} \in \mathcal{J}^{n}$ such that $m_{p}=n_{p}^{\mathbf{v}}, f_{p}(\mathbf{v})=p$ and $\beta\left(n_{p}^{\mathbf{v}}, n_{q}^{\mathbf{v}}\right) \leq 1 / 2$. As by Proposition $1, f(\mathbf{v}) \in$ $\{\bar{p} q, p \bar{q}, \bar{p} \bar{q}\}$, it follows that $f(\mathbf{v})=p \bar{q}$. We consider two cases. 
Case 1: $n_{q}^{\mathbf{v}} \geq l_{q}$. Let $\mathbf{v}^{\prime} \in \mathcal{J}^{n}$ be the voting profile arising from $\mathbf{v}$ by replacing each $\bar{p}$ by $p$. By monotonicity, the resulting decision is $f\left(\mathbf{v}^{\prime}\right)=p \bar{q}$. But $f\left(\mathbf{v}^{\prime}\right)=p q$ by Proposition 1 as $\beta\left(n_{p}^{\mathbf{v}^{\prime}}, n_{q}^{\mathbf{v}^{\prime}}\right)=\beta\left(n, n_{q}^{\mathbf{v}}\right) \geq \beta\left(n, l_{q}\right)>1 / 2$ (where the first inequality holds by $n_{q}^{\mathbf{v}}>l_{q}$ and the second by definition of $l_{q}$ ).

Case 2: $n_{q}^{\mathbf{v}}<l_{q}$. Then by Claim $2 f(\mathbf{v})=\bar{p} \bar{q}$, a contradiction since $f(\mathbf{v})=p \bar{q}$.

We have shown one inequality of Claim 3; the other one has an analogous proof.

Claim 4: For all $\mathbf{v} \in \mathcal{J}^{n}$ with $\beta\left(n_{p}^{\mathbf{v}}, n_{q}^{\mathbf{v}}\right) \leq 1 / 2$, if $n_{p}^{\mathbf{v}} \geq m_{p}$ then $f(\mathbf{v})=p \bar{q}$, and if $n_{q}^{\mathbf{v}} \geq m_{q}$ then $f(\mathbf{v})=\bar{p} q$.

Consider any $\mathbf{v} \in \mathcal{J}^{n}$ with $\beta\left(n_{p}^{\mathbf{v}}, n_{q}^{\mathbf{v}}\right) \leq 1 / 2$. Suppose for a contradiction that $n_{p}^{\mathbf{v}} \geq m_{p}$ but $f(\mathbf{v}) \neq p \bar{q}$. Then, as by Proposition $1 f(\mathbf{v}) \in\{\bar{p} q, p \bar{q}, \bar{p} \bar{q}\}$, either $f(\mathbf{v})=\bar{p} q$ or $f(\mathbf{v})=\bar{p} \bar{q}$.

Case 1: $f(\mathbf{v})=\bar{p} q$. Let $\mathbf{v}^{\prime} \in \mathcal{J}^{n}$ be the voting profile arising from $\mathbf{v}$ by replacing each $\bar{q}$ by $q$. By monotonicity, the resulting decision is $f\left(\mathbf{v}^{\prime}\right)=\bar{p} q$, whereas by Proposition $1 f\left(\mathbf{v}^{\prime}\right)=p q$ because $\beta\left(n_{p}^{\mathbf{v}^{\prime}}, n_{q}^{\mathbf{v}^{\prime}}\right)=\beta\left(n_{p}^{\mathbf{v}}, n\right) \geq \beta\left(l_{p}, n\right)>1 / 2$, where the first inequality holds because $n_{p}^{\mathbf{v}} \geq l_{p}$ (by Claim 3) and the second inequality holds by definition of $l_{p}$.

Case 2: $f(\mathbf{v})=\bar{p} \bar{q}$. By definition of $m_{p}$ there is a $\mathbf{w} \in \mathcal{J}^{n}$ such that $n_{p}^{\mathbf{w}}=m_{p}$, $f_{p}(\mathbf{w})=p$ and $\beta\left(n_{p}^{\mathbf{w}}, n_{q}^{\mathbf{w}}\right) \leq 1 / 2$. As by Proposition $1 f(\mathbf{w}) \in\{p \bar{q}, \bar{p} q, \bar{p} \bar{q}\}$, it follows that $f(\mathbf{w})=p \bar{q}$. Let $\mathbf{v}^{\prime}\left[\mathbf{w}^{\prime}\right]$ be the voting profile arising from $\mathbf{v}[\mathbf{w}]$ by replacing each $q$ by $\bar{q}$. By monotonicity, $f\left(\mathbf{v}^{\prime}\right)=\bar{p} \bar{q}$ and $f\left(\mathbf{w}^{\prime}\right)=p \bar{q}$. Now let $\mathbf{w}^{\prime \prime}$ be a voting profile arising from $\mathbf{w}^{\prime}$ by replacing $n_{p}^{\mathbf{v}^{\prime}}-n_{p}^{\mathbf{w}^{\prime}}\left(=n_{p}^{\mathbf{v}}-m_{p} \geq 0\right)$ occurrences of $\bar{p}$ by $p$. By monotonicity, $f\left(\mathbf{w}^{\prime \prime}\right)=p \bar{q}$. So, $f\left(\mathbf{w}^{\prime \prime}\right) \neq f\left(\mathbf{v}^{\prime}\right)$, a contradiction by anonymity since $\mathbf{w}^{\prime \prime}$ is a permutation of $\mathbf{v}^{\prime}$.

This shows the first implication in Claim 4 . The second one can be shown similarly.

Claim 5: $\beta\left(m_{p}, l_{q}\right), \beta\left(l_{p}, m_{q}\right)>1 / 2$.

We only show that $\beta\left(m_{p}, l_{q}\right)>1 / 2$; the other inequality is analogous. Suppose for a contradiction that $\beta\left(m_{p}, l_{q}\right) \leq 1 / 2$. So, since $\beta\left(n+1, l_{q}\right)>\beta\left(n, l_{q}\right)>1 / 2$ (by definition of $l_{q}$ ), we have $m_{p} \neq n+1$. Hence, there is a $\mathbf{v} \in \mathcal{J}^{n}$ such that $n_{p}^{\mathbf{v}}=m_{p}$ and $n_{q}^{\mathbf{v}}=l_{q}$. By Claim $4, f(\mathbf{v})=p \bar{q}$. Let $\mathbf{v}^{\prime}$ be the voting profile arising from $\mathbf{v}$ by replacing each $\bar{p}$ by $p$. By monotonicity, $f\left(\mathbf{v}^{\prime}\right)=p \bar{q}$, a contradiction since by Proposition $1 f\left(\mathbf{v}^{\prime}\right)=p q$ since $\beta\left(n_{p}^{\mathbf{v}^{\prime}}, n_{q}^{\mathbf{v}^{\prime}}\right)=\beta\left(n, l_{q}\right)>1 / 2$.

Claim 6: $f$ is given by (8).

Consider any $\mathbf{v} \in \mathcal{J}^{n}$ and $r \in\{p, q\}$. We show the equivalence (8) by distinguishing different cases. If $\beta\left(n_{p}^{\mathbf{v}}, n_{q}^{\mathbf{v}}\right)>1 / 2$, then $f(\mathbf{v})=p q$ by Proposition 1 , implying (8). If $\beta\left(n_{p}^{\mathbf{v}}, n_{q}^{\mathbf{v}}\right) \leq 1 / 2$ and $n_{r}^{\mathbf{v}} \geq m_{r}$, then (8) holds by Claim 4 . Finally, if $\beta\left(n_{p}^{\mathbf{v}}, n_{q}^{\mathbf{v}}\right) \leq 1 / 2$ and $n_{r}^{\mathbf{v}}<m_{r}$, then $f_{r}(\mathbf{v}) \neq r$ by definition of $m_{r}$, whence (8) again holds.

Proof of Theorem 8. A. First, suppose informative voting is sincere. Equivalently, for any given type $t \in \mathcal{J}$, the decision $d=t$ has maximal conditional expected utility, i.e., $\left(^{*}\right) E(u(d, S) \mid t)$ is maximal at $d=t$. Applying $\left(^{*}\right)$ with $t=p q$, we have $E(u(p q, S) \mid t) \geq E(u(\bar{p} \bar{q}, S) \mid t)$, which by Lemma 5 reduces to $\operatorname{Pr}(p q \mid t) \geq 1-\operatorname{Pr}(p q \mid t)$, i.e., to $\operatorname{Pr}(p q \mid t) \geq 1 / 2$. Using (11), one derives that $\frac{\pi_{p}}{1-\pi_{p}} \times \frac{\pi_{q}}{1-\pi_{q}} \geq C$. Now applying $\left(^{*}\right)$ with $t=p \bar{q}$, we have $E(u(p \bar{q}, S) \mid t) \geq E(u(p q, S) \mid t)$, which by Lemma 5 reduces to $1-\operatorname{Pr}(p q \mid t) \geq \operatorname{Pr}(p q \mid t)$, so that $\operatorname{Pr}(p q \mid t) \leq 1 / 2$. Using (12), one obtains $\frac{\pi_{p}}{1-\pi_{p}} \times \frac{\pi_{q}}{1-\pi_{q}} \leq$ 
A. Finally, applying (*) with $t=\bar{p} q$, we have $E(u(\bar{p} q, S) \mid t) \geq E(u(p q, S) \mid t)$, which by Lemma 5 reduces to $1-\operatorname{Pr}(p q \mid t) \geq \operatorname{Pr}(p q \mid t)$, whence $\operatorname{Pr}(p q \mid t) \leq 1 / 2$. Using (13), one derives $\frac{\pi_{p}}{1-\pi_{p}} \times \frac{\pi_{q}}{1-\pi_{q}} \leq B$. This proves all inequalities.

B. Conversely, suppose $A, B \geq \frac{\pi_{p}}{1-\pi_{p}} \times \frac{\pi_{q}}{1-\pi_{q}} \geq C$. For each given type $t \in \mathcal{J}$, one has to show $\left(^{*}\right)$. As the reader can verify using Lemma 5 and (11)-(14), if $t=p q$ then (*) follows from $\frac{\pi_{p}}{1-\pi_{p}} \times \frac{\pi_{q}}{1-\pi_{q}} \geq C$; if $t=p \bar{q}$ then $\left(^{*}\right)$ follows from $A \geq \frac{\pi_{p}}{1-\pi_{p}} \times \frac{\pi_{q}}{1-\pi_{q}}$; if $t=\bar{p} q$ then $(*)$ follows from $B \geq \frac{\pi_{p}}{1-\pi_{p}} \times \frac{\pi_{q}}{1-\pi_{q}}$; and if $t=\bar{p} \bar{q}$ then $\left(^{*}\right)$ can be derived from $A \geq \frac{\pi_{p}}{1-\pi_{p}} \times \frac{\pi_{q}}{1-\pi_{q}}$ or from $B \geq \frac{\pi_{p}}{1-\pi_{p}} \times \frac{\pi_{q}}{1-\pi_{q}}$. 